\title{
Resonant acousto-optics in the terahertz range: TO-phonon polaritons driven by an ultrasonic wave
}

\author{
E. A. Muljarov, ${ }^{*}$ R. H. Poolman, and A. L. Ivanov \\ School of Physics and Astronomy, Cardiff University, The Parade, Cardiff CF24 3AA, United Kingdom
}

(Received 21 September 2010; published 17 March 2011)

\begin{abstract}
The resonant acousto-optic effect is studied both analytically and numerically in the terahertz range where the transverse-optical (TO) phonons play the role of a mediator which strongly couples the ultrasound and light fields. A propagating acoustic wave interacts with the TO phonons via anharmonic channels and opens band gaps in the TO-phonon polariton energy dispersion that results in pronounced Bragg scattering and reflection of the incoming light. The separation in frequency of different Bragg replicas, which is at the heart of acousto-optics, allows us to study the resonant acousto-optic effect in the most simple and efficient geometry of collinear propagation of electromagnetic and ultrasonic waves. The acoustically induced energy gaps, Bragg reflection spectra, and the spatial distribution of the electric field and polarization are calculated for $\mathrm{CuCl}$ parameters, in a wide range of frequencies and intensities of the pumping acoustic wave. Our results show drastic changes in terahertz spectra of semiconductor crystals that open the way for efficient and accessible manipulation of their infrared properties by tuning the parameters of the acoustic wave.
\end{abstract}

DOI: 10.1103/PhysRevB.83.115204

PACS number(s): 71.36.+c, 43.35.Sx, 42.70.Qs

\section{INTRODUCTION}

Acousto-optics is a well established discipline and has a relatively long history which started in 1922 from a pioneering work by Leon Brillouin, ${ }^{1}$ who predicted light diffraction by an acoustic wave (AW) propagating in a medium. Ten years later this prediction received clear experimental confirmation; ${ }^{2,3}$ however, the dielectric constant modulation caused by the photoelastic effect turned out to be extremely small (of order of $10^{-5}$ ). Such a modulation leads to a similarly small light diffraction effect, which is normally seen only in the lowest diffraction order. This picture is typical for conventional, nonresonant acousto-optics. ${ }^{4}$ In contrast, resonant acoustooptics, which has been introduced very recently, ${ }^{5-8}$ deals with acoustically induced spectral changes which are comparable to the background signal. This is achieved by a resonant coupling of the incoming light to such fundamental excitations in the solid state as excitons or transverse optical (TO) phonons leading to the formation of polaritons. ${ }^{9}$ These excitations, in turn, are strongly coupled to the AW via the deformation potential (in case of excitons) or phonon anharmonicities (in case of TO phonons). This results in drastic changes of the optical properties of solid-state structures when they are exposed to an ultrasonic wave. The predicted strong resonant acousto-optic effect has been recently observed in semiconductor microcavities parametrically driven by a surface AW. ${ }^{10,11}$ A similar effect has been proposed and observed in photonic crystal structures, in which the distributed feedback cavity enhances the light-sound interaction. ${ }^{12-17}$

A traveling AW produces a propagating periodic perturbation in a solid-state structure. To some extent, such periodicity is similar to that in a photonic crystal. Thus the Bragg diffraction of light typical for photonic crystal slabs is expected also in structures with traveling modulations. In the case of an acoustically induced grating the periodic modulation propagates in space with the speed of sound. Therefore, we are dealing with a dynamical photonic crystal structure which is essentially different from conventional photonic crystals. Apart from a perfect periodicity induced by the AW, an obvious advantage of this dynamical structure is the ability to tune the Bragg lattice constant by changing the frequency of the pumping AW. A further advantage stems from a fundamental property of these dynamical structures, that the Bragg replicas, which can be spatially resolved in the far field, also acquire a spectral resolution. In this case the scheme for the detection of Bragg scattering can be greatly simplified by allowing the light and the AW to propagate collinearly, therefore, using only the spectral/temporal resolution. We employ such a scheme in the present paper assuming both light and ultrasound waves propagating in the same/opposite direction that allows us to reduce the strong-coupling theory of a three-body problem to an effective one-dimensional dynamical problem.

The purpose of this paper is to study in detail the far-infrared properties of TO-phonon polaritons driven by an ultrasonic wave, by solving (numerically) exactly a pair of Maxwell's and material macroscopic equations. $\mathrm{A} \mathrm{CuCl}$ semi-infinite crystal is taken as an example in all simulations presented in the paper. Since the TO-phonon resonant energy typically ranges from several to tens of milli-electron-volts $(20.3 \mathrm{meV}$ in $\mathrm{CuCl})$ we concentrate in this paper on polariton properties in the far infrared (terahertz) range.

We show, in particular, that owing to the anharmonic coupling to TO phonons, a coherent ultrasonic excitation gives rise to traveling periodic modulations in semiconductor structures and to dynamical Bragg scattering of electromagnetic waves. In unbound semiconductors, we describe this scattering in terms of polaritonic partial plane waves which include all important Bragg harmonics and calculate the quasienergy spectrum of such excitations. The translational invariance of the quasienergy spectrum brings into resonance different polariton states which strongly couple to each other. As a result of such anticrossing of different polariton branches, band gaps induced by the coherent AW open in the quasienergy polariton spectrum and manifest themselves as maxima in the reflection which are in turn efficiently tunable by changing parameters of the pump AW. Expanding the electromagnetic field and the TO-phonon polarization into partial polaritonic waves of unbound system, we calculate exactly (with a given accuracy) 
the full reflectivity spectra as well as frequency-selected particular Bragg replicas and their interference and analyze in detail their dependence on the AW parameters.

We have also developed a perturbative analytic approach which allows us to calculate acoustically induced band gaps and Bragg reflection spectra and analyze them in terms of phonon-assisted transitions between different TO-phonon polariton states. Comparing analytic results with the full numerical calculation of the reflection, we demonstrate that the analytical approximation works well for low and moderate excitation power of the AW. Based on the translational symmetry of the AW-driven polariton quasienergy spectrum, the analytic approach allows us to show explicitly that the acoustooptic effect is resonantly enhanced in downconverted Bragg replicas. The maximum enhancement occurs at the frequencies satisfying the Bragg condition, ${ }^{4}$ due to the polariton scattering by the AW accompanied by emission of acoustic phonons.

The paper is organized as follows. Section II presents the general formalism of the approach, introducing the macroscopic equations valid for collinear propagation of the light and ultrasound waves and their solutions in terms of plane waves. The quasienergy spectrum of bulk TO-phonon polaritons driven by an AW is calculated and analyzed in Sec. III. The vacuum-semiconductor interface is introduced in Sec. IV, where the frequency-resolved Bragg replicas and the full reflectivity spectrum are calculated, both analytically and numerically. In Sec. V, we discuss in more detail how the terahertz properties of semiconductors change with acoustic frequency, paying attention to the limits of very high and very low acoustic frequencies as well as to decay and redistribution of the electric field. Results are summarized in Sec. VI. Some important details of the analytic approach and numerical methods used in the present work are given in Appendixes A-E.

\section{FORMALISM}

The propagating AW is excited through the piezoelectric component of the electron-phonon interaction. At the same time, it couples to TO phonons via anharmonic channels. The lowest-order effect is the cubic phonon anharmonicity, which is described by the following interaction, here written in terms of phonon displacement operators: ${ }^{18,19}$

$V=\sum_{\mathbf{k} \mathbf{p}} \Phi^{(3)}(\mathbf{k}, \mathbf{p})\left(b_{\mathbf{p}}+b_{-\mathbf{p}}^{\dagger}\right)\left(b_{\mathbf{k}-\mathbf{p}}+b_{-\mathbf{k}+\mathbf{p}}^{\dagger}\right)\left(a_{-\mathbf{k}}+a_{\mathbf{k}}^{\dagger}\right)$,

where $a_{\mathbf{k}}$ and $b_{\mathbf{k}}$ are the acoustic and TO-phonon annihilation operators, respectively. In case of a propagating ultrasonic pump wave, the acoustic-phonon operators in Eq. (1) are replaced by their classical expectation values, giving

$$
V=\sigma \sum_{\mathbf{p}} b_{\mathbf{p}} b_{-\mathbf{K}+\mathbf{p}}^{\dagger} e^{i \Omega t}+\text { H.c. }
$$

where off-resonant terms are neglected (they are, however, taken into account below in the macroscopic equations). The coupling strength

$$
\sigma \propto \sqrt{I_{\mathrm{ac}}} \Phi^{(3)}
$$

is proportional to the AW amplitude ( $I_{\mathrm{ac}}$ is the acoustic intensity) and to the Fourier transform of the third-order derivative of the interatomic potential $\Phi^{(3)}$. A careful investigation ${ }^{8}$ shows that, to good approximation, any dependence on phonon wave vectors in $\Phi^{(3)}$ can be safely neglected. The operating acoustic frequencies are all limited to the linear regime of the acoustic phonon dispersion given by

$$
\Omega(K)=v_{\mathrm{s}} K,
$$

where $\mathbf{K}$ is the acoustic wavevector $(K=|\mathbf{K}|)$ and $v_{\mathrm{s}}$ is the sound velocity.

The macroscopic polarization $\mathbf{P}(\mathbf{r}, t)$ due to the TO phonons is introduced as the inverse Fourier transform of the expectation value of the TO-phonon displacement operator, $\left\langle b_{\mathbf{p}}(t)+b_{-\mathbf{p}}^{\dagger}(t)\right\rangle$. Then, using the fact that $\mathbf{D}=\varepsilon_{b} \mathbf{E}+4 \pi \mathbf{P}$, where $\mathbf{E}$ is the electric field and $\varepsilon_{b}$ is the background dielectric constant, the TO-phonon polariton in the field of a coherent pump AW is described by a system of coupled Maxwell's and material equations. ${ }^{5,8}$ In what follows, we concentrate on collinear propagation of the acoustic and light waves, normal to the semiconductor surface. In case of a semiconductor occupying half space $z>0$, the system can be effectively reduced to a one-dimensional geometry and is described by the following differential equations $(\hbar=1$ is used for simplicity of notations):

$$
\begin{aligned}
& \left(\frac{\varepsilon_{b}}{c^{2}} \frac{\partial^{2}}{\partial t^{2}}-\frac{\partial^{2}}{\partial z^{2}}\right) E(z, t)=-\frac{4 \pi}{c^{2}} \frac{\partial^{2}}{\partial t^{2}} P(z, t) \\
& {\left[\frac{\partial^{2}}{\partial t^{2}}+2 \gamma \frac{\partial}{\partial t}+\omega_{t}^{2}+4 \sigma \omega_{t} \cos (K z-\Omega t)\right] P(z, t)} \\
& =\frac{\varepsilon_{b} \omega_{R}^{2}}{4 \pi} E(z, t)
\end{aligned}
$$

where $\omega_{t}$ and $\gamma$ are, respectively, the frequency and the damping of bulk dispersionless TO phonons, $\omega_{R}$ is the Rabi frequency which characterizes the coupling strength between light and TO phonons. Equations (5) and (6) generalize the famous polaritonic Hopfield model ${ }^{9}$ by adding the interaction with the acoustic field. They can be derived with the help of Heisenberg equations of motion from the Hamiltonian of TO-phonon polaritons. ${ }^{20,21}$ An essential difference to the conventional polariton model is the appearance of an oscillating term in Eq. (6), which originates from the TO-phonon coupling to the coherent AW described by Eq. (2).

We solve Eqs. (5) and (6) in the semiconductor region in terms of partial plane waves. Due to the oscillating pump acoustic field, the electric field and polarization of each partial wave can be expanded into the following Fourier series

$$
\begin{aligned}
& E(z, t)=e^{i(k z-\omega t)} \sum_{n} E_{n} e^{i n(K z-\Omega t)}, \\
& P(z, t)=e^{i(k z-\omega t)} \sum_{n} P_{n} e^{i n(K z-\Omega t)},
\end{aligned}
$$

where $\omega$ and $k$ are, respectively, the leading frequency and wave vector of the partial wave of the electromagnetic field. The acoustically induced perturbation is periodic in both time and space and thus it is formally required to make a double Fourier transform. However, as shown in Appendix A, this leads to linearly dependent combinations of plane waves, so 
that the double Fourier transform of $E$ and $P$ is effectively reduced to the form of Eqs. (7) and (8). We substitute them into Eqs. (5) and (6) and equate coefficients at the same exponentials. This results in the following matrix eigenvalues problem:

$$
\begin{gathered}
{\left[(k+n K)^{2}-\frac{\varepsilon_{b}}{c^{2}}(\omega+n \Omega)^{2}\right] E_{n}=\frac{4 \pi}{c^{2}}(\omega+n \Omega)^{2} P_{n},} \\
{\left[\omega_{t}^{2}-2 i \gamma(\omega+n \Omega)-(\omega+n \Omega)^{2}\right] P_{n}} \\
+2 \sigma \omega_{t}\left(P_{n+1}+P_{n-1}\right)=\frac{\varepsilon_{b} \omega_{R}^{2}}{4 \pi} E_{n} .
\end{gathered}
$$

We solve the set of coupled linear equations [Eqs. (9) and (10)] and find the polariton dispersion $\omega(k)$ and the corresponding eigenvectors $E_{n}$ and $P_{n}$ which are the amplitudes of the partial plane waves of the TO-phonon polariton propagating in a semiconductor modulated by the AW.

The matrix eigenvalue problem formulated in Eqs. (9) and (10) is, in general, complex, and the values of the light frequency $\omega$ and/or momentum $k$ can be complex even if the damping $\gamma$ is neglected. Here we distinguish two different physical problems: (i) a modified polariton quasienergy spectrum where for any given real value of the polariton momentum $k$ we find the complex polariton energy $\omega$, as done in Sec. III below; (ii) the far infrared properties of the acoustically driven TO-phonon polaritons, such as reflection, scattering, and transmission. Therefore, we are dealing with the real frequency $\omega$ of the incoming light. In case (ii), considered in Sec. IV in detail, the wave vector $k$ is complex valued as it describes propagation and attenuation of the polariton waves in the medium.

In all numerical simulations done in the present work we use the following parameters of bulk $\mathrm{CuCl}:{ }^{22} \mathrm{TO}$-phonon energy $\omega_{t}=20.28 \mathrm{meV}$, Rabi splitting $\omega_{R}=14.53 \mathrm{meV}$, low-temperature phonon damping ${ }^{23,24} \gamma=0.2 \mathrm{meV}$ (at $T=$ 5-10 K), background dielectric constant $\varepsilon_{b}=5.2$, and sound velocity $v_{s}=2.02 \times 10^{3} \mathrm{~m} / \mathrm{s}$. The coupling constant $\sigma$, one of the important parameters of the model, scales with the acoustic intensity according to Eq. (3), with the value of $\sigma=1 \mathrm{meV}$ corresponding ${ }^{8}$ to the acoustic intensity $I_{\mathrm{ac}}=14.8 \mathrm{~kW} / \mathrm{cm}^{2}$.

\section{QUASIENERGY SPECTRUM OF ACOUSTICALLY DRIVEN TO-PHONON POLARITONS}

\section{A. Eigenfrequencies}

In unbound semiconductors, the polariton quasimomentum $k$ is a good quantum number which takes real values. For nonzero damping $\gamma$, the polariton eigenfrequencies, in turn, are always complex. Let us, however, concentrate on propagating solutions and disregard, for the moment, energetic band gaps where propagation is not allowed. Then, assuming $\gamma=0$, all eigenfrequencies also become real. This slightly idealized picture of undamped polaritons in unbound semiconductors will help us, however, to understand the basic properties of the polariton dispersion modified by the AW.

Treating $k$ as a real parameter, we solve the eigenvalue problem [Eqs. (9) and (10)], finding polariton eigenfrequencies $\omega=\tilde{\omega}_{\lambda}(k)$ which correspond to the energy bands $\lambda=1,2, \ldots$ separated by acoustically induced band gaps. Technically, we truncate Eqs. (9) and (10) at $-n_{\max } \leqslant n \leqslant n_{\max }$, introducing the matrix dimension $M=2 n_{\max }+1$. The system of two $M \times$ $M$ matrix equations, containing nonlinear dependence both on frequency and wave vector is reduced, in Appendix B, to the following linear matrix eigenvalue problem

$$
\overrightarrow{\mathbb{W}} \overrightarrow{\mathbb{X}}=\tilde{\omega} \overrightarrow{\mathbb{X}}
$$

solved by means of complex matrix diagonalization, where $\hat{\mathbb{W}}$ is an $\tilde{\omega}$-independent $4 M \times 4 M$ hypermatrix and $\overrightarrow{\mathbb{X}}$ is a $4 M$ hypervector containing the Fourier components of the electric field and polarization, defined in Eqs. (B8) and (B9), respectively. Note that the extra factor of 4 in the matrix dimension $4 M$ comes from two different sources. The existence of two branches in the bare polariton spectrum, the so-called upper polariton (UP) and lower polariton (LP) branches provide a factor of 2. The fact that all eigenfrequencies come in pairs ${ }^{25}$ $\left(\tilde{\omega}_{\lambda}\right.$ and $\left.-\tilde{\omega}_{\lambda}^{*}\right)$ provides the remaining factor of 2 .

Clearly, the quasienergy spectrum $\tilde{\omega}_{\lambda}(k)$ has the following translational property valid within the same branch $\lambda$ :

$$
\tilde{\omega}_{\lambda}(k+s K)=\tilde{\omega}_{\lambda}(k)+s \Omega,
$$

where $s$ is an integer. The corresponding eigenfunctions are also invariant in a way that, for the same $\lambda$,

$$
E_{n}(k+s K)=E_{n+s}(k),
$$

and a similar expression is valid for $P_{n}$.

The translationally invariant polariton spectrum $\tilde{\omega}_{\lambda}(k)$ is shown in Fig. 1 as a function of the polariton momentum

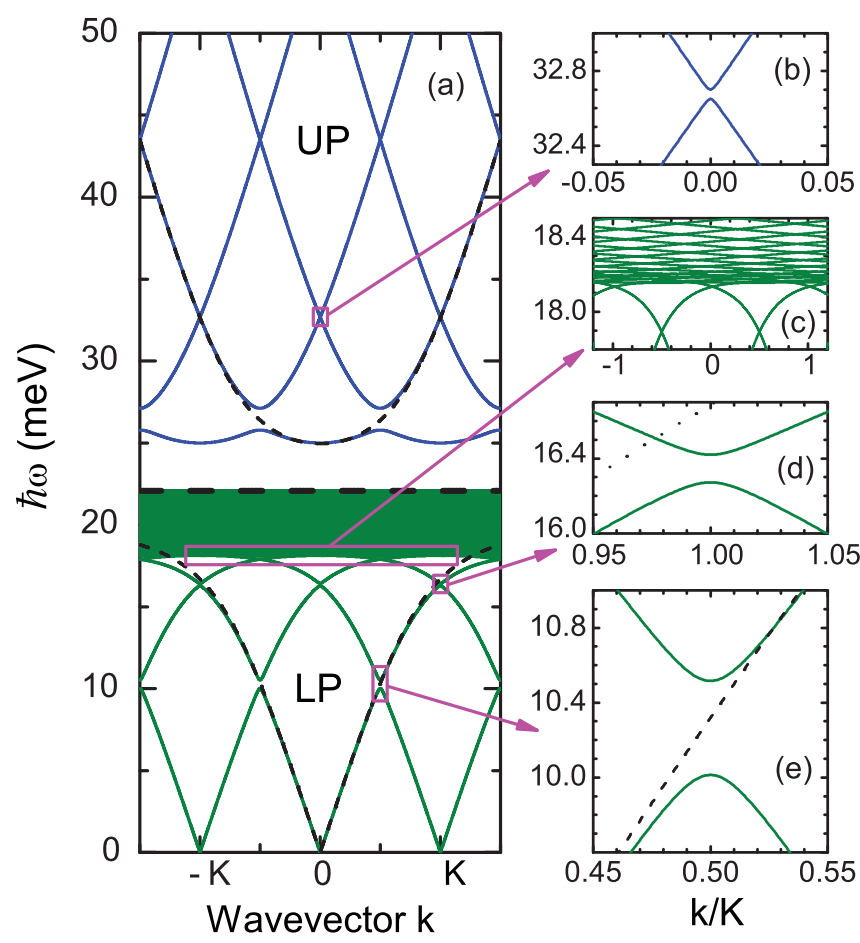

FIG. 1. (Color online) Quasienergy spectrum of UP (blue) and LP (green) branches of undamped TO-phonon bulk polaritons driven by an AW with $v=\Omega /(2 \pi)=100 \mathrm{MHz}\left(K=0.3109 \mu \mathrm{m}^{-1}\right)$ and $\sigma=1 \mathrm{meV}$. The spectrum without $\mathrm{AW}, \omega=\omega^{0}(k)$, is given by thin dashed curves. The thick dashed horizontal line shows an artificial cutoff in the quasicontinuum of LP states. Panels (b)-(e) highlight particular areas in the spectrum. 
$k$ (measured in units of the acoustic wavevector $K$ ). Due to the spatial and temporal periodicity imposed by the pumping $\mathrm{AW}$, the bare polariton spectrum (dashed curves) is folded into the first Brillouin zone and then translational shifted, both horizontally and vertically, into all other Brillouin zones. The coupling of polaritons to the AW opens gaps in the folded polariton spectrum, just in the center and on the borders of the Brillouin zone [see Figs. 1(b), 1(d), and 1(e)]. These gaps are acoustically induced spectral regions where polariton propagation is not allowed and thus they crucially affect the terahertz properties of the polaritons, such as light reflection and Bragg scattering. The positions of the gaps are determined mainly by the frequency $\Omega$ (or wave vector $K$ ) of the pumping $\mathrm{AW}$, whereas their widths strongly depend on both the wave vector $K$ and the intensity (coupling strength $\sigma$ ) of the AW, as discussed in detail in the next section.

In the polariton bands, which are the regions where polariton propagation is allowed, the modified spectrum deviates from the bare one. This is illustrated in Figs. 1(d) and 1(e). This deviation become dramatic close to and above the TO-phonon resonant energy. Unlike the bare polariton dispersion in which the LP branch is bounded by the TO-phonon frequency, the AW-induced branches of the quasienergy spectrum, even those which originate from the LP branch, can be found at any frequencies. Thus, they are, in principle, unbound. They form a sort of a quasicontinuum of polariton states detailed in Fig. 1(c), which is due to the acoustically induced anticrossing and level repulsion. The optical strength of these quasicontinuum states, however, drops quickly with energy and contributes to the reflectivity only at the frequencies around the low-energy edge of the Restrahlen band.

\section{B. Acoustically induced band gaps}

The band gaps are spectral regions forbidden for polariton propagation; therefore, one can anticipate an enhancement of reflection at the frequencies of incoming light close to or inside the gaps. The analytic calculation of acoustically induced band gaps, which we shown in this section, is particularly important for the understanding of this enhancement and other terahertz properties of acoustically driven polaritons, considered later in Sec. IV.

The tridiagonal form of the matrix in Eq. (10) allows us to evaluate the widths of the energy gaps analytically. Expressing $P_{n}$ from the diagonal Eq. (9) and substituting it into Eq. (10) results in

$$
\alpha_{n} D_{n} E_{n}-\sigma\left(\alpha_{n+1} E_{n+1}+\alpha_{n-1} E_{n-1}\right)=0,
$$

with

$$
\begin{gathered}
D_{n}(k, \omega) \\
=\frac{1}{2 \omega_{t}}\left[\omega_{t}^{2}-2 i \gamma(\omega+n \Omega)-(\omega+n \Omega)^{2}-\frac{\omega_{R}^{2}}{\alpha_{n}(k, \omega)}\right] \\
\alpha_{n}(k, \omega)=\frac{c^{2}}{\varepsilon_{b}}\left(\frac{k+n K}{\omega+n \Omega}\right)^{2}-1
\end{gathered}
$$

Equation (14) can be solved recursively. Introducing $\xi_{n}=$ $E_{n} / E_{n+1}$, it is transformed to

$$
\xi_{n}=\frac{\sigma \alpha_{n+1}}{\alpha_{n} D_{n}-\sigma \alpha_{n-1} \xi_{n-1}} .
$$

With a truncation $E_{-N-1}=E_{1}=0$, Eq. (14) is solved up to the $N$ th order. The ratio $\sigma / D_{n} \sim 2 \sigma / \omega_{t}$ serves as a smallness parameter in our perturbation approach which breaks down when the frequency $\omega$ approaches either the TO-phonon resonance $\omega_{t}$ or the bottom of the UP branch, $\sqrt{\omega_{t}^{2}+\omega_{R}^{2}}$.

The above truncation is equivalent to the following boundary conditions added to the recursive equation (17):

$$
\xi_{-N-1}=1 / \xi_{0}=0 .
$$

Taking the first boundary condition $\xi_{-N-1}=0$ as a staring point, we find $\xi_{-N}, \xi_{-N+1}$, and so on, ending up with the relation $\alpha_{0} D_{0}=\sigma \alpha_{-1} \xi_{-1}$, in which the second boundary condition is used. The above procedure turns out to be particularly useful for a perturbative approach to the resonant acousto-optic effect discussed in Sec. IV D, where the Bragg replicas are calculated analytically in lowest orders. In the present section we evaluate perturbatively only the acoustically induced band gaps, again assuming $\gamma=0$.

In the $N=0$ case, Eqs. (17) and (18) result in a single equation,

$$
D_{0}(k, \omega)=0,
$$

which yields the bare polariton dispersion $\omega=\omega^{0}(k)$ consisting of the LP and UP branches (see dashed curves in Fig. 1), with no effect from the AW. The acoustically induced gaps appear in the polariton spectrum in first and higher perturbation orders at specific values of the polariton wave vector given (neglecting the acoustic frequency $\Omega$ ) by the famous Bragg condition

$$
k_{N}=N K / 2, \quad N=1,2, \ldots,
$$

which corresponds to centers and borders of different Brillouin zones (obviously all of them can be translated into the first Brillouin zone). The energy positions of the gaps, $\omega_{N}$, are given (in zeroth order) by the bare polariton dispersion,

$$
\omega_{N}=\omega^{0}\left(k_{N}\right),
$$

or, in terms of Eq. (19), can be found by solving the equation $D_{0}\left(k_{N}, \omega_{N}\right)=0$. The widths of the gaps, $\Delta_{N}$, are evaluated in lowest order in Appendix $\mathrm{C}$, with the following result:

$$
\begin{aligned}
& \Delta_{1}=\sigma S\left(\omega_{1}\right), \\
& \Delta_{2}=\sigma^{2} \frac{S\left(\omega_{2}\right)}{\left|D_{-1}\left(k_{2}, \omega_{2}\right)\right|}, \\
& \Delta_{3}=\sigma^{3} \frac{S\left(\omega_{3}\right)}{D_{-1}^{2}\left(k_{3}, \omega_{3}\right)}, \\
& \Delta_{4}=\sigma^{4} \frac{S\left(\omega_{4}\right)}{D_{-1}^{2}\left(k_{4}, \omega_{4}\right)\left|D_{-2}\left(k_{4}, \omega_{4}\right)\right|}, \\
& \ldots,
\end{aligned}
$$

where

$$
S(\omega)=\frac{2 \omega \omega_{t} \omega_{R}^{2}}{\left(\omega^{2}-\omega_{t}^{2}\right)^{2}+\omega_{t}^{2} \omega_{R}^{2}} .
$$

The gap $\Delta_{N}$ opens in the $N$ th perturbation order as a result of anticrossing of different polariton dispersion branches. The anticrossings occur due to the translational invariance of the spectrum which brings into resonance states originating from 
negative- $k$ and positive- $k$ parts of the bare polariton dispersion, for example, by a translational shift in the $(k, \omega)$ plane of the states in the negative- $k$ part of the dispersion by the vector $(N K, N \Omega)$, that is, by the same integer of the wave vector and the frequency of the AW. In other words, the $N$ th gap opens due to the $N$-acoustic phonon transition, induced by the pumping AW, which couples two points in the bare dispersion $\omega^{0}(k)$ having the opposite values of $k$ as given by the Bragg condition [Eq. (20)]. As a result, the width of the gap $\Delta_{N}$ is proportional to $\sigma^{N}$ (and to $I_{\mathrm{ac}}^{N / 2}$ ). The form factor $S(\omega)$ grows by increasing the TO-phonon component and reducing the light component of the polariton; hence, the gaps become wider. In fact, since TO phonons play the role of a mediator between the light and acoustic fields (in our model, these two fields do not interact directly), and thus the more the component of TO phonons in the polariton field, the stronger is the resonant acousto-optic effect.

Figure 2 presents a comparison of the analytical and numerical calculations of the band gaps at a moderate acoustic intensity $I_{\mathrm{ac}}=2.4 \mathrm{~kW} / \mathrm{cm}^{2}(\sigma=0.4 \mathrm{meV})$. The analytic results for the widths of the two lowest band gaps, $\Delta_{1}$ and $\Delta_{2}$ (lines), are in excellent agreement with the full numerical calculation (symbols), practically in the whole range of the acoustic frequencies and both in the UP and LP parts of the spectrum, as seen from Fig. 2. At smaller detuning which
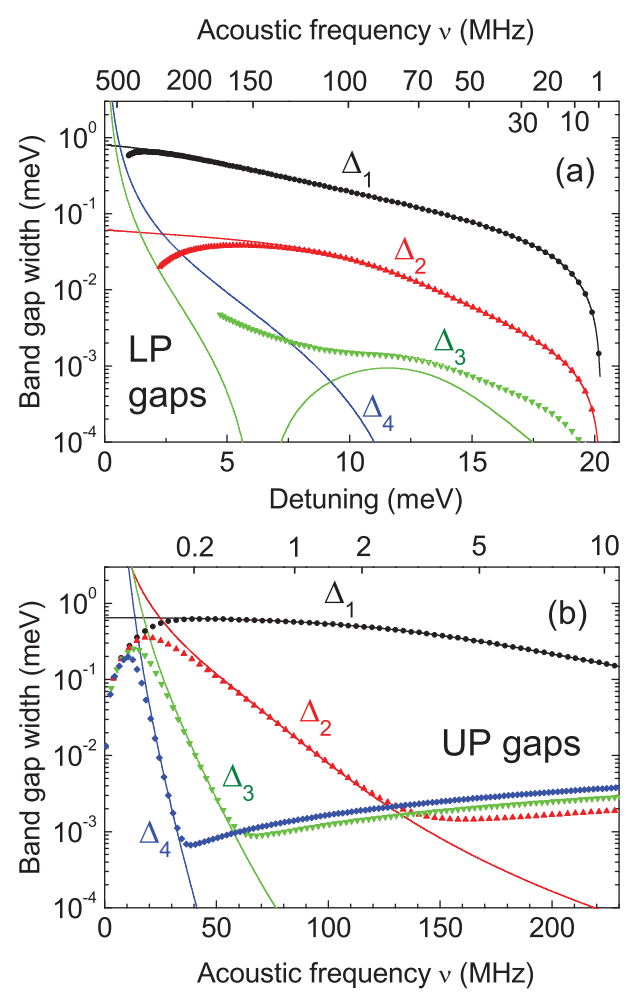

FIG. 2. (Color online) (a) Analytical (curves) and numerical (symbols) calculation of the acoustically induced band gaps in the LP branch vs the acoustic frequency $v=\Omega /(2 \pi)$ (top axis) and the detuning $\omega_{t}-\omega_{1}$ (bottom axis). (b) Energy gaps in the UP branch vs acoustic frequency (bottom axis) and the detuning $\omega_{1}-\sqrt{\omega_{t}^{2}+\omega_{R}^{2}}$ (top axis). In cases (a) and (b), $\omega_{1}$ is the energy position of the lowest gap, either in the LP (a) or in the UP (b) part of the spectrum. The coupling strength $\sigma=0.4 \mathrm{meV}$, and the TO-phonon damping $\gamma=0$. corresponds to higher (lower) acoustic frequency in the LP (UP) branch, the agreement gets worse because the analytical model, which is based on the assumption that the detuning is large compared to the coupling strength, breaks down in this case. At the same time, higher-order band gaps like $\Delta_{3}$ and $\Delta_{4}$ are not well reproduced by the analytics. This can be explained by the fact that the present analytic approach neglects the acoustic frequency $\Omega$, assuming that it is small compared both to the light frequency and to the energy gaps (for details, see Appendix C ). This, however, is not the case of the third- and fourth-order band gap, as their widths fall into sub- $\mu \mathrm{eV}$ range and thus become comparable to or even smaller than $\Omega$.

\section{BRAGG REFLECTION OF ELECTROMAGNETIC WAVES}

\section{A. Polariton quasimomenta}

The quasienergy spectrum and the band gaps discussed in detail in Sec. III can be probed in experiments by a monochromatic electromagnetic wave with frequency $\omega$. In this case, the boundary between the semiconductor and the vacuum becomes an essential part of the system, so that the momentum across the surface is no longer conserved. However, for the given frequency $\omega$ of incoming light, the polariton waves can have only specific values of the complex momentum, $k=\tilde{\kappa}_{j}(\omega)$. They are known in the literature as forced harmonic solutions. ${ }^{26}$ The complex quasimomenta $\tilde{\kappa}_{j}(\omega)$ can be found as eigenvalues of the same equations [(9) and (10)]. In this case we solve the inverse problem to Sec. III A: The complex quasimomenta $\tilde{\kappa}_{j}(\omega)$ are found as eigenvalues. The electric field and polarization of the propagating polariton waves are the corresponding normalized eigenvectors, $E_{n j}$ and $P_{n j}$, respectively, where an extra index $j=0, \pm 1, \pm 2, \ldots$ is added in order to distinguish different partial waves. For each frequency, Maxwell's boundary conditions determine a unique combination of the polariton partial waves and finally the reflection and transmission properties which are discussed in subsequent sections. The partial waves themselves, however, are not fully independent. For any integer number $s$, the wave vectors and amplitudes of the partial waves obey the translational relations [cf. with Eqs. (12) and (13)]

$$
\begin{gathered}
\tilde{\kappa}_{j+s}(\omega)=\tilde{\kappa}_{j}(\omega-s \Omega)+s K, \\
E_{n, j+s}(\omega)=E_{n+s, j}(\omega-s \Omega), \\
P_{n, j+s}(\omega)=P_{n+s, j}(\omega-s \Omega),
\end{gathered}
$$

following from the spatiotemporal periodicity of the system pumped by an AW, periodic in time and space.

The quasimomenta $\tilde{\kappa}_{j}$ are calculated in the following way. The infinite-matrix problem Eqs. (9) and (10) is again truncated to $-n_{\max } \leqslant n \leqslant n_{\max }\left(M=2 n_{\max }+1\right)$. The amplitude $P_{n}$ is expressed from Eq. (9) and substituted into Eq. (10). The latter equation is then linearized with respect to $\tilde{\kappa}$, as done in Appendix B, and brought to the form

$$
\hat{\mathbb{V}} \overrightarrow{\mathbb{Y}}=\tilde{\kappa} \overrightarrow{\mathbb{Y}}
$$

with a $2 M \times 2 M$ hypermatrix $\hat{\mathbb{V}}$ and a $2 M$ hypervector $\overrightarrow{\mathbb{Y}}$ defined in Eqs. (B19) and (B20), respectively. There is now 


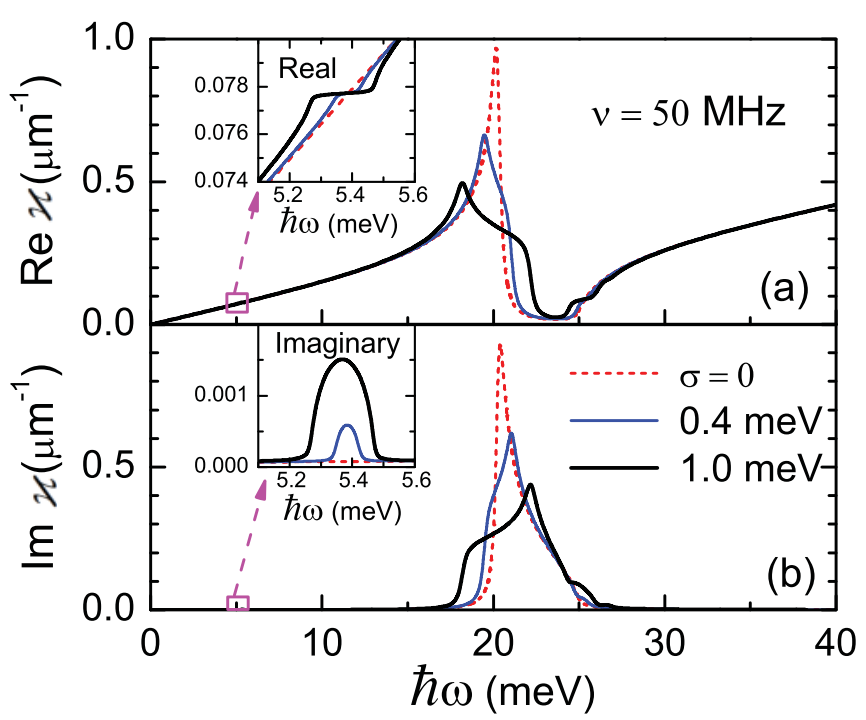

FIG. 3. (Color online) (a) Real part and (b) imaginary part of the polariton quasimomentum $\varkappa(\omega)$ calculated with (blue thin and black thick curves) and without AW (red dashed curves). The insets highlight particular regions corresponding to the lowest acoustically induced gap. The TO-phonon damping $\gamma=0.2 \mathrm{meV}$ and the AW frequency $v=50 \mathrm{MHz}\left(K=0.1555 \mu \mathrm{m}^{-1}\right)$.

only one extra factor of 2 in the matrix dimensions $2 M$. This is due to the symmetry $\tilde{\kappa} \rightarrow-\tilde{\kappa}$ of the initial eigenvalue problem. ${ }^{25}$ However, there is no more clear separation of the eigenvalues into LP and UP branches.

Technically, we calculate the eigenvalues $\tilde{\kappa}_{j}$ for quite big matrices (with $M=161$, so that $-80 \leqslant j \leqslant 80$ ) for different values of $\omega$ and then select only the central root,

$$
\varkappa(\omega) \equiv \tilde{\kappa}_{j=0}(\omega),
$$

that is, the root which satisfies the equation $\varkappa(0)=0$. The central root is chosen as best calculated. In fact, it is the most distant solution from the boundaries of the truncated array, so that any distortions of the spectrum due to such truncation are minimized in the center of the array. We then use this central root $\varkappa$ to improve calculation of all other wave vectors $\tilde{\kappa}_{j}(j \neq 0)$ exploiting the translational property [Eq. (24)] and making a linear interpolation Eq. (B21) between neighboring frequency points.

Both the real and the imaginary parts of the central root $\varkappa$ are shown in Fig. 3, in the presence of AW and without it. Apart from very strong changes in the polariton momentum slightly below the TO-phonon resonant frequency (20.3 meV) and in the whole Restrahlen band above it, tiny changes occur near the acoustically induced gaps and grow with increasing coupling strength $\sigma$ (see the insets to Fig. 3). These small features, however, are very important for the infrared properties of the system and thus we consider them in more detail.

The real part of the quasimomentum [Fig. 4(a), top panel], if it were calculated for $v=100 \mathrm{MHz}$, would reproduce the quasienergy spectrum shown in Fig. 1(e) for zero damping. The only difference would be the horizontal connection line in Fig. 4(a). However, the results shown in Figs. 3 and 4 are calculated for half that acoustic frequency, which has moved the gap to energies nearly half that in Fig. 1(e). The energy gap

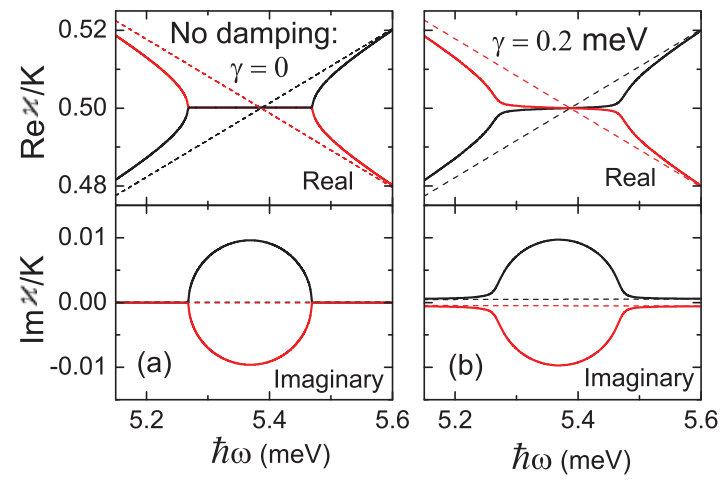

FIG. 4. (Color online) Real and imaginary parts of the polariton quasimomentum $\varkappa(\omega)$ calculated around the acoustically induced gap (a) for zero damping $\gamma=0$ and (b) for finite damping $\gamma=0.2 \mathrm{meV}$, with (solid lines) and without AW (dashed lines). Black and red (gray) colors correspond to positive- $k$ unshifted and negative- $k$ one TA-phonon shifted polariton dispersion branches, respectively. The AW frequency $v=50 \mathrm{MHz}$.

is due to the acoustically induced strong coupling between the two bare polariton branches crossed in the center of Fig. 4(a) (see dashed lines). The real part of the momentum shows the typical anticrossing behavior while the imaginary part, strictly absent at $\gamma=\sigma=0$, demonstrates a pronounced band gap. Polariton waves for which the frequencies fall in the gap region propagate only into a limited area due to their finite decay in space, which is determined by the imaginary part of the wave vector. With finite damping (which is still smaller than the band-gap width), the situation does not change much, but the imaginary part of the wave vector remains finite everywhere in this case [see Fig. 4(b)].

\section{B. Bragg replicas: Numerical approach}

Let us now consider the effects of reflection, transmission and Bragg scattering due to a semiconductor-vacuum boundary at $z=0$. In the semiconductor area $(z>0)$, the electric field and TO-phonon polarization are superpositions of partial polaritonic waves,

$$
\begin{gathered}
E(z, t)=\sum_{j} A_{j} E_{j}(z, t)=\sum_{n j} A_{j} E_{n j} e^{i\left(\tilde{\kappa}_{j}+n K\right) z-i(\omega+n \Omega t)}, \\
P(z, t)=\sum_{n j} A_{j} P_{n j} e^{i\left(\tilde{\kappa}_{j}+n K\right) z-i(\omega+n \Omega t)}
\end{gathered}
$$

where $E_{n j}$ and $P_{n j}$ are normalized eigenvectors and $\tilde{\kappa}_{j}$ are eigenvalues described and calculated in Sec. IV A. In the vacuum region $(z<0)$, the electric field is given by

$$
E(z, t)=e^{i q_{0} z} e^{-i \omega t}+\sum_{n} r_{n} e^{-i q_{n} z} e^{-i(\omega+n \Omega) t},
$$

where $q_{n}=(\omega+n \Omega) / c$ is the wave vector and $r_{n}$ is the amplitude of the outgoing Bragg replica $n$. The amplitude $r_{n}$ is normalized to the unity amplitude of the incoming light wave, which is described by the first term on the right-hand side of Eq. (31). Coefficients $A_{j}$, which stand for the amplitudes of the partial waves, are found from Maxwell's boundary conditions. These require continuity of the electric and magnetic fields, 
$E(z, t)$ and $H(z, t)$, across the interface $z=0$. Applying the boundary conditions to the electric field [Eqs. (29) and (31)] and to the magnetic field calculated via $\partial H / \partial t=c \partial E / \partial z$ results in the set of equations

$$
\begin{gathered}
e^{-i \omega t}+\sum_{n} r_{n} e^{-i(\omega+n \Omega) t}=\sum_{n j} A_{j} E_{n j} e^{-i(\omega+n \Omega) t}, \\
e^{-i \omega t}-\sum_{n} r_{n} e^{-i(\omega+n \Omega) t}=\sum_{n j} A_{j} E_{n j} \frac{\tilde{\kappa}_{j}+n K}{q_{n}} e^{-i(\omega+n \Omega) t},
\end{gathered}
$$

which have to be satisfied at any time $t$. Thus, we equate coefficients of the same exponentials, which then leads to a set of linear algebraic equations for $r_{n}$ and $A_{j}$ :

$$
\begin{gathered}
\delta_{n, 0}+r_{n}=\sum_{j} A_{j} E_{n j}, \\
\delta_{n, 0}-r_{n}=\sum_{j} A_{j} E_{n j} \frac{\tilde{\kappa}_{j}+n K}{q_{n}} .
\end{gathered}
$$

Equations (32) and (33) are solved numerically, using a truncation $-n_{B} \leqslant n \leqslant n_{B}$ with the total number of Bragg replicas $N_{B}=2 n_{B}+1$. Normally, the number of Bragg replicas $N_{B}$ required for an accurate calculation of the reflectivity spectra is much smaller than the matrix dimension $M$ used in the calculation of the polariton quasimomentum $\varkappa(\omega)$, $N_{B} \ll M$. For example, to reach the accuracy $10^{-7}$ in the calculation of $\mathrm{CuCl}$ reflectivity using the $\mathrm{AW}$ parameters $\sigma=2 \mathrm{meV}$ and $v=100 \mathrm{MHz}$, one needs to take only $N_{B}=21$ and $M=81$ into account.

\section{Reflectivity spectra}

In our geometry, the light and the AW propagate collinearly and thus there is no way to have any spatial selection of the Bragg replicas. However, each Bragg replica has it own unique temporal evolution as can be seen in Eq. (31). Thus, using a frequency-selective technique one can measure the intensity of the $n$th Bragg replica in the reflected light,

$$
R_{n}=\left|r_{n}\right|^{2}
$$

The $n$th replica carries the frequency $\omega+n \Omega$; it is downconverted (upconverted) with respect to the frequency $\omega$ of the incoming light for negative (positive) $n$.

Without such frequency selection, the total but still spectrally resolved Bragg reflection can be measured. Due to the different time evolution of different Bragg signals, there can be two opposite ways to measure the reflectivity. One is fully incoherent; in this case the temporal resolution $\tau$ of the spectrometer is insufficient to see the interference fringes between different Bragg replicas. In other words, the interference terms fully vanish in the measured electric field. This happens when $\Omega \tau \gg 1$, so that $\tau$ falls into a nanosecond range. In this case, the total Bragg reflectivity is given by

$$
R=\sum_{n=-n_{B}}^{n_{B}}\left|r_{n}\right|^{2} .
$$

Alternatively, the spectrometer can have enough temporal resolution and then the opposite limit $\Omega \tau \ll 1$ is realized,
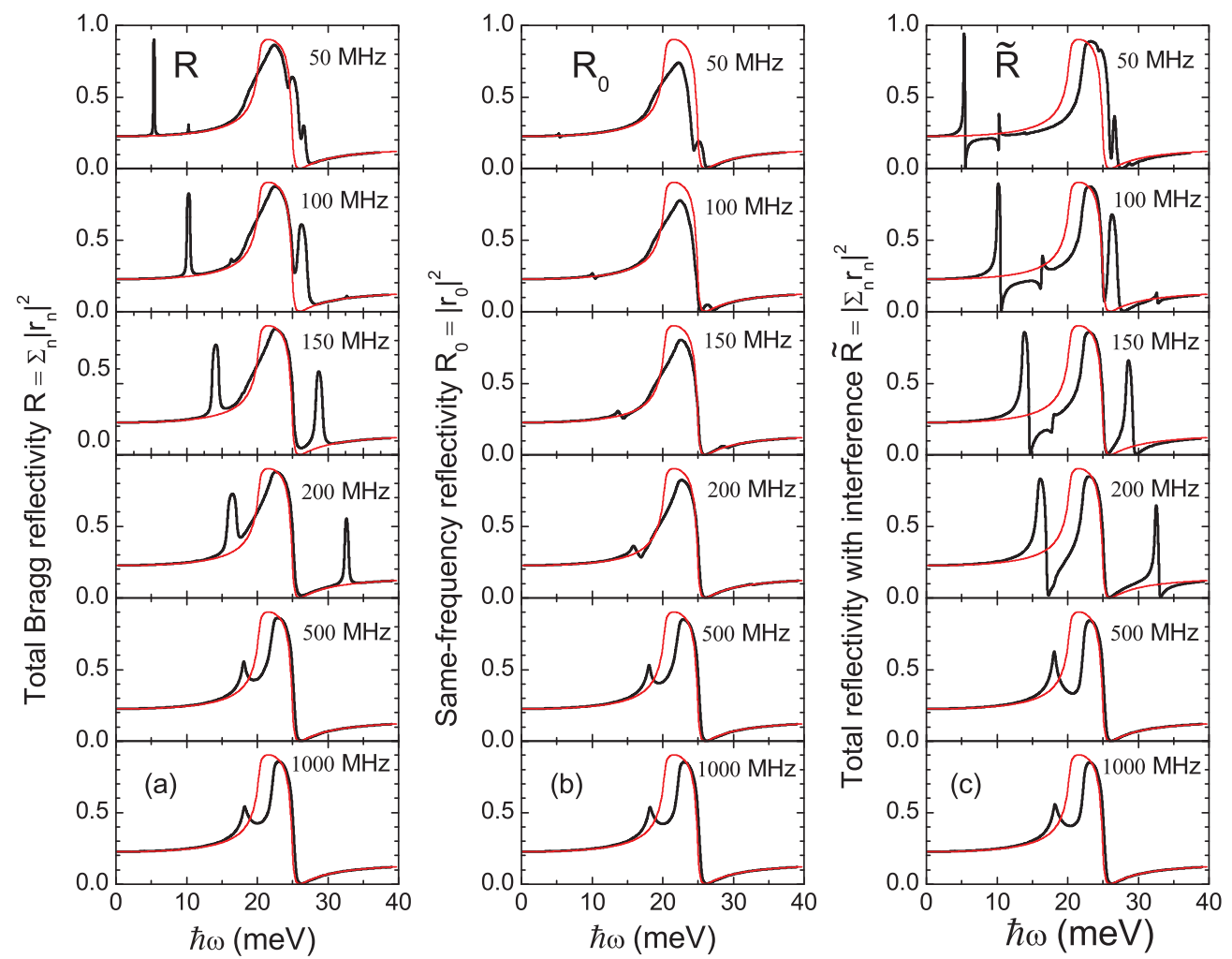

FIG. 5. (Color online) (a) Incoherent and (c) coherent total Bragg reflectivity calculated for $\sigma=1 \mathrm{meV}$ and different acoustic frequencies, without and with interference terms, respectively. (b) The same-frequency central Bragg replica. The bare reflection spectrum of damped TO phonons (with no AW) is shown by red (gray) thin curves. 
making the total Bragg reflectivity fully coherent:

$$
\widetilde{R}=\left|\sum_{n=-n_{B}}^{n_{B}} r_{n}\right|^{2} .
$$

Such a regime is achievable when the spectrometer resolution is in the sub-nanosecond or even picosecond range.

We show in Fig. 5 both the coherent and the incoherent signals, $\widetilde{R}(\omega)$ and $R(\omega)$, in comparison with the zeroth replica $R_{0}(\omega)$. Note that $R_{0}$ is the only component which survives in the absence of any acoustic pumping; for $\sigma=0$ it is shown in Fig. 5 by a red (gray) curve, the same in all panels. All other spectra shown in Fig. 5 by black curves are calculated for $\sigma=1 \mathrm{meV}$. They reveal drastic changes compared to the bare $(\sigma=0)$ spectrum. By increasing the acoustic frequency from $50 \mathrm{MHz}$ to $1 \mathrm{GHz}$, a strong spike in the reflectivity $R$, which is associated with the acoustically modified LP branch moves toward the Restrahlen band, gets thicker, and finally saturates into a cusplike feature close to the bare TO-phonon transition energy. Well below the TO-phonon energy, the spike is exclusively due to the first-order acoustically induced band gap discussed in Sec. III B. Its thickness is given either by the band gap width $\Delta_{1}$ [see Eq. (22)] or by the phonon damping, whichever is greater. A similar spike which comes from the UP branch is well resolved above the Restrahlen band, for the acoustic frequencies between 100 and $300 \mathrm{MHz}$. The coherent Bragg reflectivity $\widetilde{R}$ has similar properties; however, the spectral changes are enhanced considerably by pronounced dispersive features which appear due to the strong interference effect.

At higher acoustic frequencies, the contribution of the central Bragg replica, $R_{0}$, becomes dominant and all other replicas vanish as $v \rightarrow \infty$, so that all three spectra $R(\omega)$, $R_{0}(\omega)$, and $\widetilde{R}(\omega)$ finally coincide. This happens because the light wavelength becomes large compared to the acoustic wavelength and thus the AW modulation of the semiconductor structure is not resolved by the light field. The acoustically modulated semiconductor works in this case as an effective homogeneous medium with a modified $\omega$-dependent dielectric constant. The cusplike structure in the reflectivity which evolves from the dispersive (N-shaped) feature in $R_{0}$ [see Fig. 5(b)] can be understood as a manifestation of the band gaps accumulating around the TO-phonon frequency. Indeed, according to Eqs. (20) and (21), at large values of the acoustic wavevector $K$, there must be a plenty of band gaps stacked together around $\omega_{t}$.

In this high-frequency limit, there are no qualitative changes in the reflectivity when the coupling to the AW increases, as is clear from Fig. 6(b), where the reflectivity $R\left(R \approx R_{0}\right)$ is shown for different values of the coupling strength $\sigma$. Indeed, in this case only the strength of the cusp, its splitting from the Restrahlen band, and the transparency of the latter grow with $\sigma$. In the opposite case of small acoustic frequencies, the increasing coupling strength does lead to qualitatively new features in the spectrum. In particular, new peaks corresponding to the second and third acoustically induced band gaps, both in the UP and in the LP branches, grow up in $v=50 \mathrm{MHz}$ spectra, as shown in Fig. 6(a).

The influence of the first- and higher-order band gaps on different Bragg replicas is analyzed in more detail in Fig. 7. The
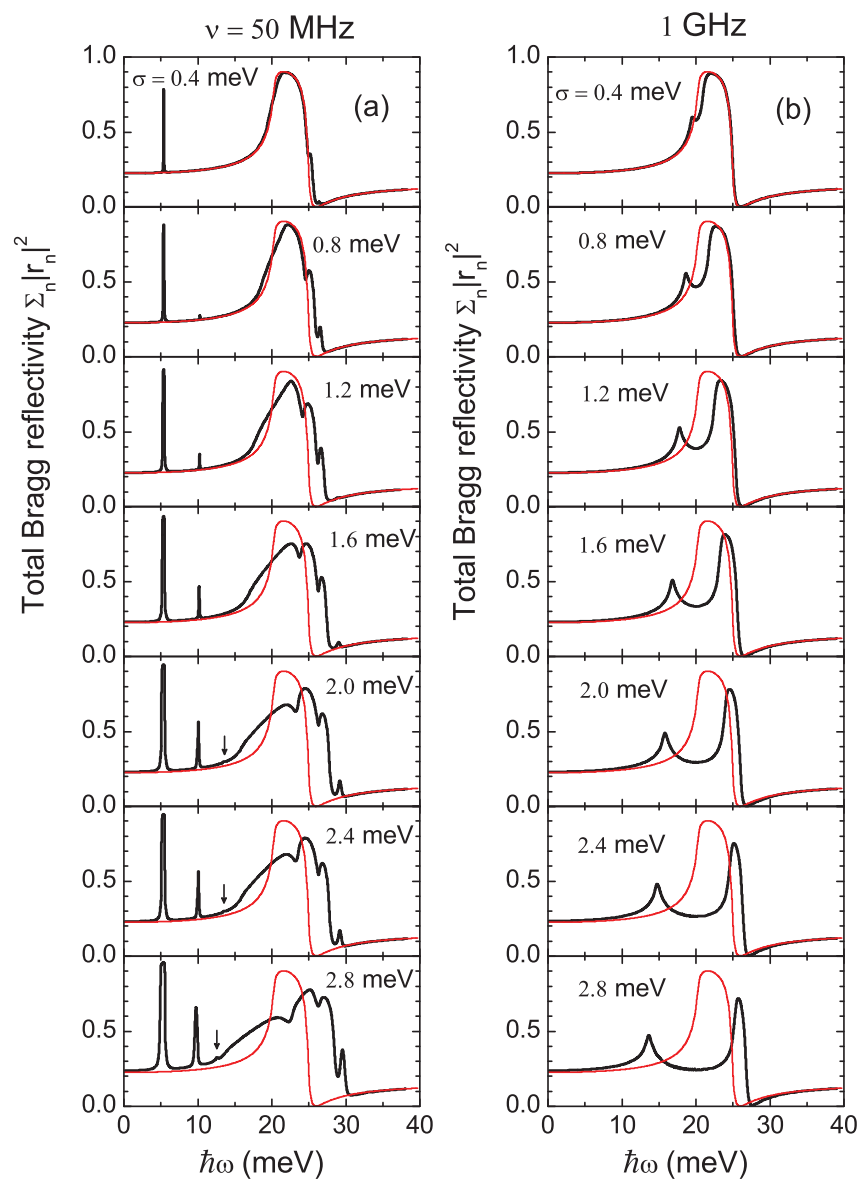

FIG. 6. (Color online) Total Bragg reflectivity $R(\omega)$ calculated for the acoustic frequency $v=\Omega /(2 \pi)=50 \mathrm{MHz}$ (a) and $1 \mathrm{GHz}$ (b) and for different values of the coupling strength $\sigma$ (acoustic intensity $I_{\mathrm{ac}}$ ). Small vertical arrows in panel (a) point at the spike growing due the third-order band gap. The bare reflection spectrum of damped TO phonons is shown by red (gray) thin curves.

top panels display the polariton quasimomentum dispersion, similar to those shown in Figs. 3 and 4. The Bragg condition is illustrated in Fig. 7 by gray horizontal lines (separated by $K / 2$ ) and by arrows all having the same length equal to $K$. The main spikes in the full reflectivity spectrum $R(\omega)$, which are shown in the bottom plots, appear exactly at the energies of the first-order band gaps. These spikes are due to onephonon transitions between positive- $\varkappa$ and negative- $\varkappa$ parts of the polariton dispersion $\varkappa(\omega)$. This effect, which is already discussed above in terms of the bare polariton dispersion $\omega^{0}(k)$ (see Figs. 1 and 4), is now demonstrated taking into account the realistic acoustically modified dispersion $\varkappa(\omega)$. With increasing acoustic frequency $\Omega$ and, consequently, increasing acoustic wavevector $K$, the same Bragg condition can only be satisfied at a higher frequency. Thus, the band gaps and the spikes are blueshifted, at the same time getting wider due to a larger TO-phonon component. This is visualized by two- and three-times-longer arrows in Figs. 7(b) and 7(c). Below the Restrahlen band, these arrows point to one-phonon transitions that occur, respectively, at nearly two- and three-times-larger frequencies. The band gaps are more pronounced at larger $\Omega$ and manifest themselves as longer and flatter plateaus in the dispersion [see Fig. 7(c), top panel]. From the practical 
(a) $\Omega /(2 \pi)=50 \mathrm{MHz}$
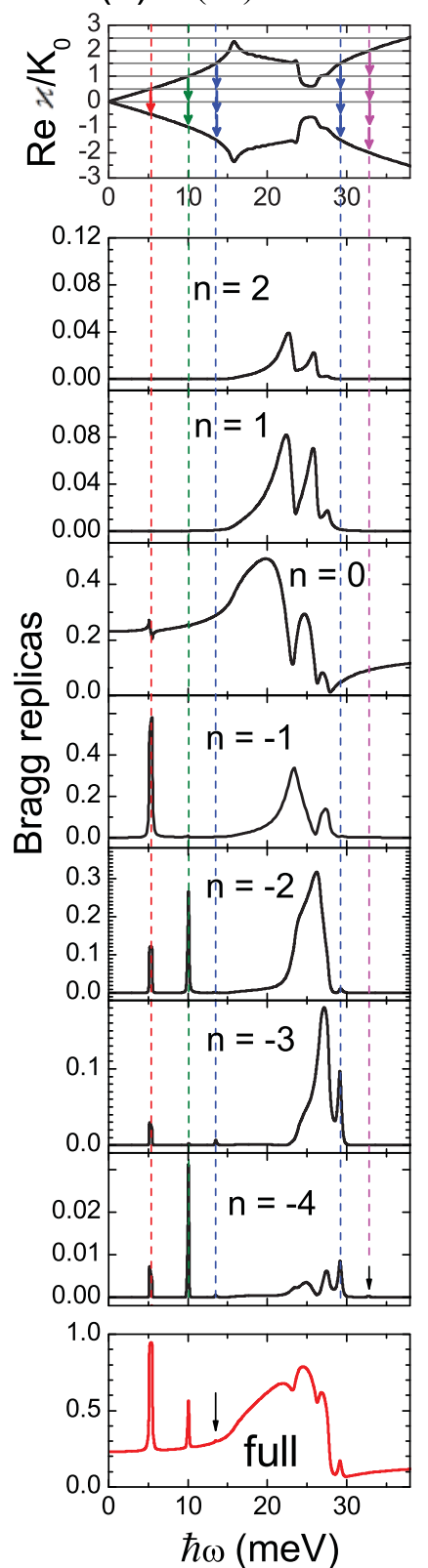

(b) $100 \mathrm{MHz}$
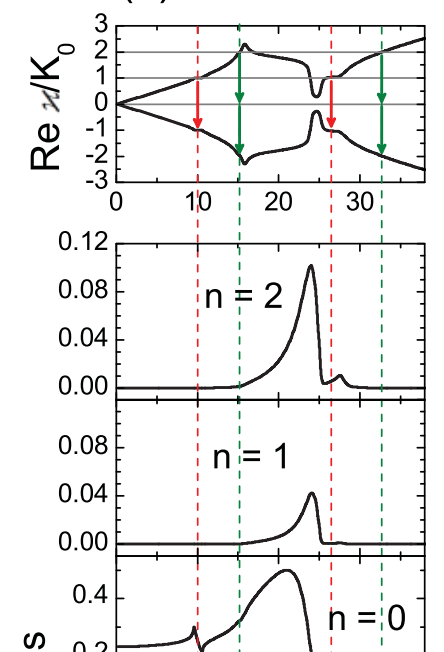

(c) $150 \mathrm{MHz}$
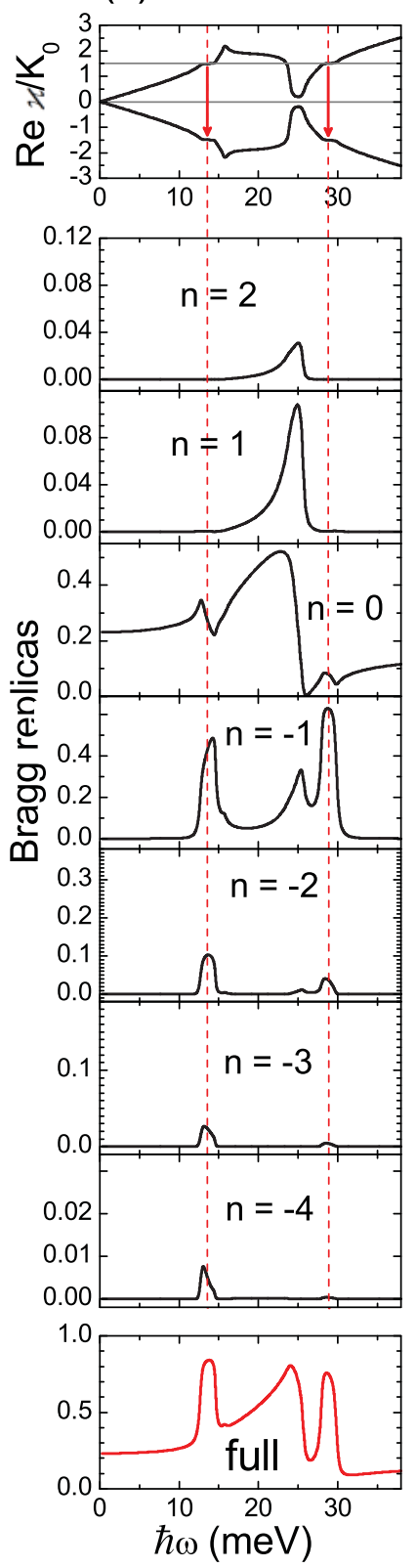

FIG. 7. (Color online) Acoustically modified polariton dispersion of damped TO phonons (top panels), contributions of different Bragg replicas $\left|r_{n}\right|^{2}$ with $n=2,1,0,-1,-2,-3$, and -4 , as well as the total reflectivity spectrum $R(\omega)=\sum_{n}\left|r_{n}\right|^{2}$ (bottom panels), calculated for $\sigma=2 \mathrm{meV}$ and $v=\Omega /(2 \pi)=50 \mathrm{MHz}$ (a), $100 \mathrm{MHz}$ (b), and $150 \mathrm{MHz}$ (c). Arrows and vertical lines indicate positions of the band gaps due to $N$-phonon dressed transitions. The acoustic wavevector takes the values of (a) $K=K_{0}$, (b) $K=2 K_{0}$, and (c) $K=3 K_{0}$, where $K_{0}=0.1555 \mu \mathrm{m}^{-1}$.

viewpoint, the effects demonstrated in Figs. 5-7 are nothing else than a very efficient way of changing the terahertz properties of semiconductor structures simply by tuning the frequency and the intensity of the pumping AW.

The $N$-phonon transitions (with $N=2,3$, and 4) are shown in Fig. 7 by a series of $N$ arrows of different color. They are responsible for higher-order acoustically induced band gaps and for new spikes, which appear in the reflectivity when $\sigma$ increases (see also Fig. 6). In particular, Fig. 7(a) demonstrates that the spikes due to second-, third-, and fourthorder gaps show up in the Bragg replicas $n=-2,-3$, and
-4 , respectively. The higher-order Bragg replicas, however, accumulate the contribution of all lower- $N$ phonon transitions in higher orders. The higher-order effects of lower- $N$ gaps are sometimes even stronger than the contribution of the same $N=|n|$ gap to the Bragg replica $r_{n}$; see, for example, $r_{-3}$ and $r_{-4}$ replicas in Fig. 7 at the frequencies $\omega \approx \omega_{1}$ and $\omega_{2}$. This effect is discussed in more detail in Sec. IV D.

The incoming light beam excites polaritons with positive values of the quasimomentum $\varkappa>0$, and then these polaritons are scattered by the AW to the negative- $\varkappa$ part of the polariton dispersion. Such scattering is accompanied by the emission 
of acoustic phonons and consequently by downconversion in frequency. These downconverted polariton waves correspond to negative- $n$ components of the electric field, both in semiconductor and in vacuum [see Eqs. (29) and (31)]. That is why all spikes in the reflection corresponding to the AW-induced band gaps appear only in Bragg replicas having negative $n$ and no such features can be seen when $n$ is positive.

By increasing the coupling strength $\sigma, N$-phonon transitions responsible for the band gaps' reflectivity spikes become increasingly dressed by higher-order transitions. In particular, the $N$ th-order gap is formed due to multiple processes of simultaneous emission of $N+s$ phonons and absorption of $s$ phonons, where $s$ can be any integer. In the present calculation, however, with the parameters used throughout the paper, this number never exceeds, in reality, $|s|=20$, even for very large acoustic intensities (up to $\sigma=3 \mathrm{meV}$ ).

\section{Bragg replicas: Analytic approach}

At low acoustic intensities (small $\sigma$ ), the Bragg replicas can be calculated using the perturbative approach introduced in Sec. III B. In this approach, the $N$ th perturbation order describes the acousto-optic effect with participation of $N$ phonons (no higher-order dressing is included), and in particular the opening of the gaps $\Delta_{N}$ due to $N$-phonon transitions. Below we calculate acousto-optic effect in each $N$ th Bragg replica in its lowest ( $N$ th) order, calculating both upconverted $r_{+N}$ and downconverted $r_{-N}$ replicas, and show explicitly that the effect is resonantly enhanced only in the downconverted replicas $r_{-1}, r_{-2}$, etc.

The perturbation-theory result for the $N$ th Bragg replicas has the following recursive form (for derivation, see Appendix D):

$$
\begin{gathered}
r_{ \pm N}=-\sum_{s=1}^{N} A_{ \pm(N-s)} E_{ \pm s} \frac{\beta_{ \pm s}-\beta_{0}}{1+\beta_{0}} \\
A_{ \pm N}=-\sum_{s=1}^{N} A_{ \pm(N-s)} E_{ \pm s} \frac{1+\beta_{ \pm s}}{1+\beta_{0}}, \\
E_{ \pm N}=\sigma^{N} \frac{\alpha_{0}}{\alpha_{ \pm N}} \prod_{s=1}^{N} \frac{1}{D_{ \pm s}}
\end{gathered}
$$

where $D_{n}(\omega, k)$ are defined in Eq. (15),

$$
\alpha_{n}=\left(\frac{k+n K}{p}\right)^{2}-1, \quad \beta_{n}=\frac{k+n K}{q}
$$

(we have neglected here any dependence on $\Omega$ ), $q=\omega / c$, and $p=\sqrt{\varepsilon_{b}} q$. The starting $N=0$ values in the recursion are given by

$$
r_{0}=\frac{1-\beta_{0}}{1+\beta_{0}}, \quad A_{0}=\frac{2}{1+\beta_{0}}, \quad E_{0}=1 .
$$

To a first approximation, the quasimomentum $k$ in the above equations is taken in zeroth order, that is, is calculated according to the bare polariton dispersion

$$
k=k^{0}(\omega)
$$

which satisfies Eq. (19) and is inverse to $\omega=\omega^{0}(k)$. Using this bare dispersion relationship, $D_{0}(\omega, k)=0$, the functions $D_{n}(\omega, k)$ are simplified to

$$
\alpha_{n} D_{n}=\frac{\omega_{R}^{2}}{2 \omega_{t}} \frac{(k+n K)^{2}-k^{2}}{k^{2}-p^{2}},
$$

where any dependence on $\Omega$ is neglected.

The explicit form of Eq. (43) allows us to analyze the resonances seen in Bragg replicas. Since $D_{n}$ stands in the denominator of the electric field components Eq. (39), $r_{n}$ have maxima when $\alpha_{n} D_{n}$ is minimized. This happens when $(k+n K)^{2} \approx k^{2}$, leading to a Lorentzian spectral line shape peaked at the Bragg condition,

$$
\operatorname{Re}(k)=-\frac{n K}{2},
$$

and having the widths proportional to $\operatorname{Im}(k)$ which at this point is fully determined by the TO-phonon damping $\gamma$. The incoming light excites polaritons with positive wave vectors $\operatorname{Re}(k)>0$; thus, the Bragg condition is fulfilled only for negative $n<0$, so that the peaks in the reflectivity are observed in the negative (downconverted) Bragg replicas only.

The amplitudes of the three lowest-order Bragg replicas have the following explicit form:

$$
\begin{gathered}
r_{-1}=\sigma \frac{K}{q} \frac{2}{\left(1+\beta_{0}\right)^{2}} \frac{\alpha_{0}}{\alpha_{-1} D_{-1}}, \\
r_{-2}=\sigma^{2} \frac{K}{q} \frac{2}{\left(1+\beta_{0}\right)^{2}} \\
\times\left[\frac{2 \alpha_{0}}{\alpha_{-2} D_{-2} D_{-1}}-\frac{1+\beta_{-1}}{1+\beta_{0}} \frac{\alpha_{0}^{2}}{\alpha_{-1}^{2} D_{-1}^{2}}\right], \\
r_{-3}=\sigma^{3} \frac{K}{q} \frac{2}{\left(1+\beta_{0}\right)^{2}} \\
\times\left[\frac{3 \alpha_{0}}{\alpha_{-3} D_{-3} D_{-2} D_{-1}}-\frac{3+2 \beta_{-1}+\beta_{-2}}{1+\beta_{0}}\right. \\
\left.\times \frac{\alpha_{0}^{2}}{\alpha_{-2} \alpha_{-1} D_{-2} D_{-1}}+\left(\frac{1+\beta_{-1}}{1+\beta_{0}}\right)^{2} \frac{\alpha_{0}^{3}}{\alpha_{-1}^{3} D_{-1}^{3}}\right] .
\end{gathered}
$$

The analytic result Eqs. (45)-(47) is demonstrated in Fig. 8(a) in comparison with the full numerical calculation; both are done for a moderate coupling strength $\sigma=0.4 \mathrm{meV}$. The agreement is good everywhere except the spikes, in which only positions are reproduced well [see a zoomed area in the inset of Fig. 8(a)]. Indeed, with purely Lorentzian peaks in the spectra, the magnitudes of the replicas sometimes exceed unity, which results in fully unphysical reflectivity. This artifact, however, is removed below and the spectrum is refined by taking into account the acoustically modified polariton dispersion. For positive values of $n$, the replicas are shown in Fig. 8(b) in order to demonstrate that no spikes at the energies of AWinduced band gaps are seen either in analytical or in numerical calculation.

The first downconverted Bragg replica $r_{-1}$ has a spike at the frequency $\omega_{1}$ of the lowest acoustically induced energy gap [see Eq. (21)]. The spike corresponds to a one-phonon transition between polariton states in the positive- $k$ and 

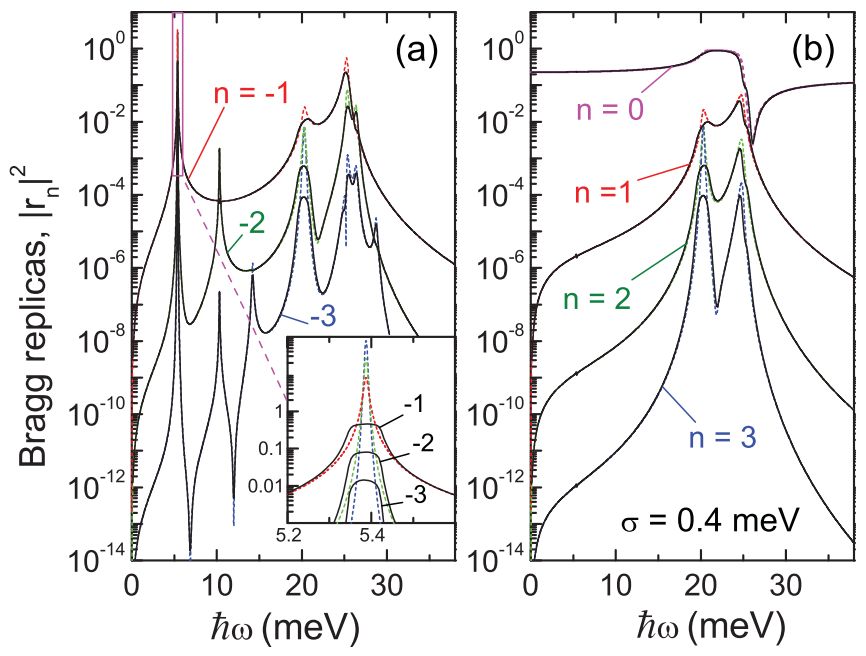

FIG. 8. (Color online) Bragg replicas $\left|r_{n}\right|^{2}$ calculated numerically (black lines) and analytically (red, green, blue, and magenta dashed lines) for (a) $n=-1,-2,-3$, and (b) $n=1,2,3$, and 0 . Parameters used in both calculations: coupling strength $\sigma=0.4 \mathrm{meV}$, acoustic frequency $v=50 \mathrm{MHz}$, TO-phonon damping $\gamma=0.2 \mathrm{meV}$. The inset zooms in on the spectral region of the first acoustically induced band gap and spikes in the Bragg reflection due to one-phonon transitions.

negative- $k$ branches of the dispersion, as shown in Fig. 7 and discussed earlier in Sec. IV C. Note that although we concentrate here mainly on the LP part of the spectrum, similar spikes can be seen also in the spectral region of the UP branch.

In the second replica $r_{-2}$, resonances appear at the energies of both first and second band gaps, $\omega_{1}$ and $\omega_{2}$. The first term in square brackets in Eq. (46) describes a composite two-phonon process with a virtual intermediate state having only one phonon emitted. That is why the first term in Eq. (46) shown in Fig. 9 by a dashed red line is peaked at both energies $\omega_{1}$ and $\omega_{2}$. The final state has the dominant role and so the resonance at $\omega \approx \omega_{2}$ is much stronger. The second term shown by blue (dotted) line in Fig. 9 has only one peak at $\omega \approx \omega_{1}$ (in the LP part of the spectrum) as it describes a one-phonon contribution in second order. This one-phonon process is the only component of the first-order Bragg replica $r_{-1}$. Surprisingly, its dominant role is well seen in $r_{-2}$ as well (see the sum of both terms, the black solid line in Fig. 9). This physical picture helps us to understand similar features in higher Bragg replicas. In particular, the first term in Eq. (47) is due to a three-phonon transition having as intermediate (virtual) states those with one and two phonons emitted. The last term is the one-phonon process taken into account in third order, and the middle term is a mixed contribution of both one and two phonon-assisted transitions. Consequently, the resonances in $r_{-3}$ occur at $\omega \approx \omega_{1}, \omega_{2}$, and $\omega_{3}$ and are due to different processes with participation of three acoustic phonons.

Let us now refine the analytic calculation in order to get rid of the artificially high analytic reflectivity which is detailed in the inset in Fig. 8(a). To do this, we simply replace the bare polariton dispersion $k=k^{0}(\omega)$ in Eqs. (40) and (43) with the acoustically modified dispersion $k=\varkappa(\omega)$ calculated in Sec. IV A [see Eq. (28)]. Consequently, a modified Bragg

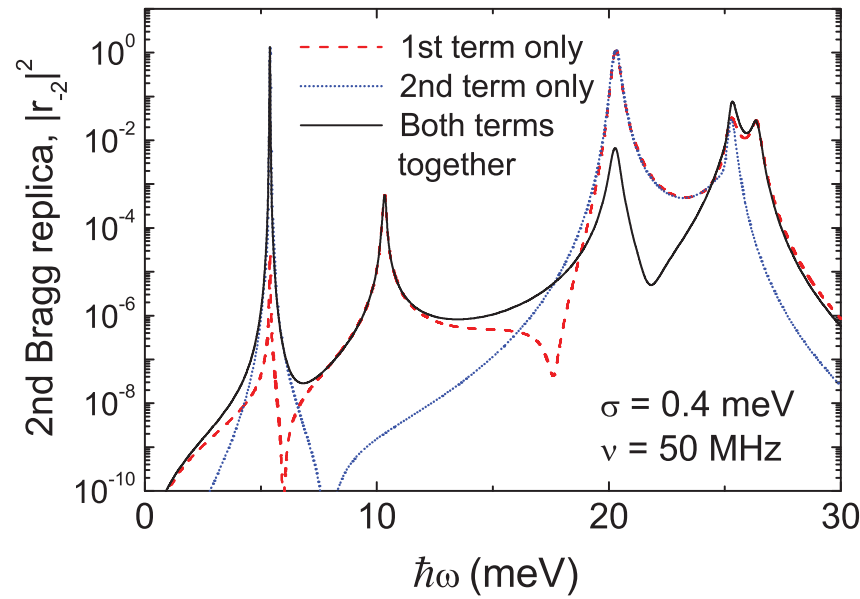

FIG. 9. (Color online) The second Bragg replica $\left|r_{-2}\right|^{2}$ calculated analytically according to Eq. (46): the first term only (red dashed line), the second term only (blue dotted line), and both terms together (black solid line). All parameters are the same as in Fig. 8.

condition which follows from Eq. (43) and determines the acousto-optic resonant energies $\omega_{n}$ becomes

$$
\operatorname{Re}\left[\varkappa\left(\omega_{|n|}\right)\right]=-\frac{n K}{2}
$$

[cf. with Eqs. (44) and (21)]. The refined Bragg condition [Eq. (48)] has been used already in our analysis of the spectra shown in Fig. 7. From the viewpoint of the perturbation theory developed in the present section, the calculation of the modified dispersion $\varkappa(\omega)$ can intrinsically take into account a large number of higher perturbation orders. Thus, it is not fully consistent with the calculation of $r_{n}$ limited to the lowest-order $|n|$ only. From the practical viewpoint, however, such a refinement works perfectly well for sufficiently narrow resonances, as can be seen in Fig. 10(a) in which the spectral lines are shown by the solid red and green (gray) curves in comparison with full calculation (black curves). Both the height and the width of the spikes are reproduced well. The narrow and unrealistically high Lorentzian lines shown by the dashed curves are fully determined by the damping $\gamma$. In the refined analytic calculation, the linewidth is determined by the band-gap width, which is properly incorporated into the modified dispersion $\varkappa(\omega)$. A slight difference between the numerical and the refined analytical calculations is due to the fact that for such a moderate acoustic intensity $(\sigma=0.4 \mathrm{meV})$, higher-order corrections (with $s>|n|$ ) to the Bragg replicas $r_{n}$ not included in Eqs. (37)-(39) are already perceptible. They become crucial in case of much wider gaps in the UP part of the spectrum, see Fig. 10(b), where the improvement due to the dispersion refinement is rather minor.

Finally, a calculation of the $n=0$ Bragg replica, which has been done up to now only in zeroth order, can be further improved by inclusion of second-order corrections:

$$
r_{0}=\frac{1-\beta_{0}}{1+\beta_{0}}+\sigma\left(\frac{K}{q}\right)^{2} \frac{4}{\left(1+\beta_{0}\right)^{3}} \frac{\alpha_{0}^{2}}{\alpha_{1} D_{1} \alpha_{-1} D_{-1}} .
$$

Clearly, this expression contains resonant features due to the $D_{-1}$ in the second term. However, unlike the higher-order replicas which exhibit the Lorentzian line shape, this resonant 


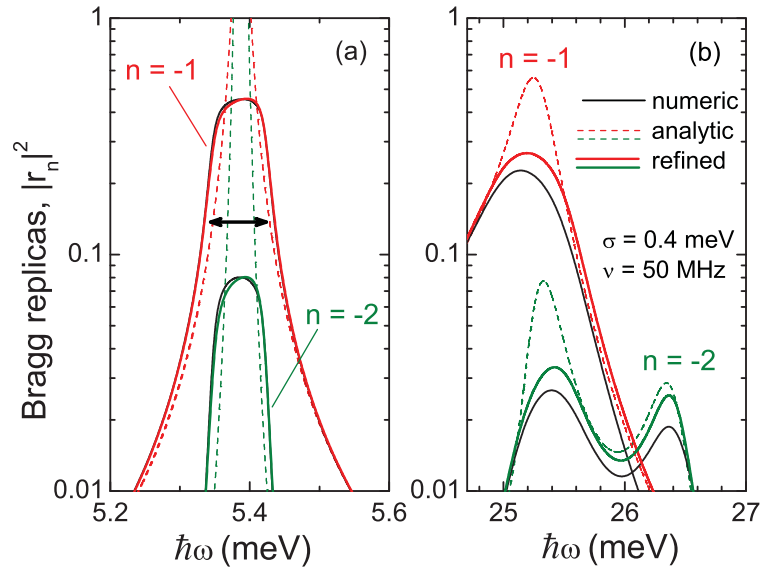

FIG. 10. (Color online) Bragg replicas $\left|r_{-1}\right|^{2}$ and $\left|r_{-2}\right|^{2}$ calculated analytically with [red and green (gray) solid lines] and without (dashed lines) refinement of the polariton dispersion. The full numerical calculation is shown by black solid lines. All parameters are the same as in Fig. 8. The double-side arrow shows the width of the first acoustically induced gap calculated via Eq. (22).

term is added to the background reflection [Eq. (41)]. Thus, $\left|r_{0}\right|^{2}$ has instead a more pronounced dispersive behavior, due to the mixing of the first and second terms in Eq. (49). Such dispersive features manifest themselves as N-shaped resonances clearly seen in Fig. 5(b) and become more and more prominent with increasing acoustic frequency (see also $n=0$ replicas in Fig. 7).

\section{DISCUSSION}

\section{A. Low and high acoustic frequency limits}

The terahertz properties of semiconductors and their changes due to varying acoustic frequency $\Omega$ have been already discussed in Sec. IV C. In particular, Fig. 5(a) has demonstrated an efficient manipulation of the spectral properties, changing both the positions of the spikes and their widths simply by tuning $\Omega$. Moreover, in the limiting case of very large $\Omega$, the wavelength of the spatial modulation caused by the AW turns out to be much smaller than the wavelength of the propagating light, so that the modulation does not produce any diffraction. As a result, all acoustically induced spectral changes are concentrated in the central Bragg replica $R_{0}$ [cf. Figs. 5(a) and $5(\mathrm{~b})$ ], while the contribution of all higher replicas vanishes at $\Omega \rightarrow \infty$. This is more clearly demonstrated in Fig. 11, where the lower panels show diminishing contribution of the higher $(|n|>0)$ Bragg replicas to the total reflection. The results are summarized in Fig. 12 where the spectral area of the higher replicas is plotted against $\Omega$. This spectral area is calculated according to

$$
\int_{-\infty}^{\infty} \sum_{n \neq 0}\left|r_{n}(\omega)\right|^{2} d \omega
$$

Surprisingly, a similar effect takes place in the opposite limit of low acoustic frequencies. Although the reduction of the contribution of higher replicas in this case is not very well illustrated by Fig. 11, and technically this limit is hard to

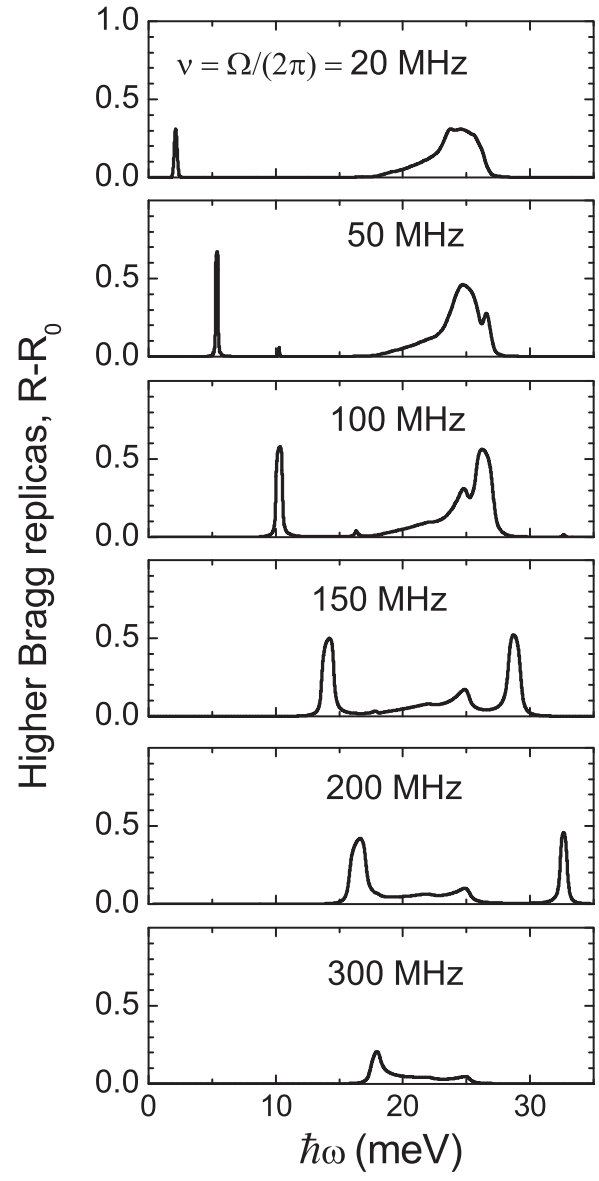

FIG. 11. The total contribution of higher Bragg replicas, $\sum_{n \neq 0}\left|r_{n}\right|^{2}$, for the coupling strength $\sigma=1 \mathrm{meV}, \gamma=0.2 \mathrm{meV}$, and different acoustic frequencies.

achieve with the present numerical method, an overall trend is such that the spectral area is getting smaller and possibly vanishes at $\Omega \rightarrow 0$, as seen in Fig. 12. In this case, the shortperiod acoustic modulations work again as a homogeneous dielectric medium, which can be be characterized by an effective (modified) dielectric constant. In a sense, this picture is similar to the effective mass approximation for electrons, valid in bulk semiconductors as well as in short-period

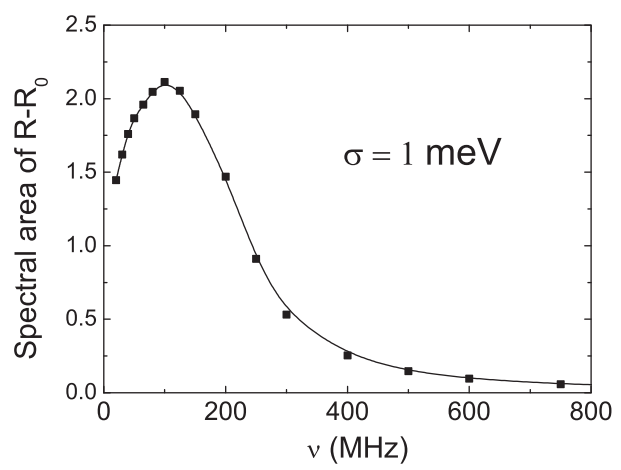

FIG. 12. The spectral area of higher Bragg replicas, calculated according to Eq. (50) for $\sigma=1 \mathrm{meV}, \gamma=0.2 \mathrm{meV}$, and different values of the acoustic frequency $v=\Omega /(2 \pi)$. 


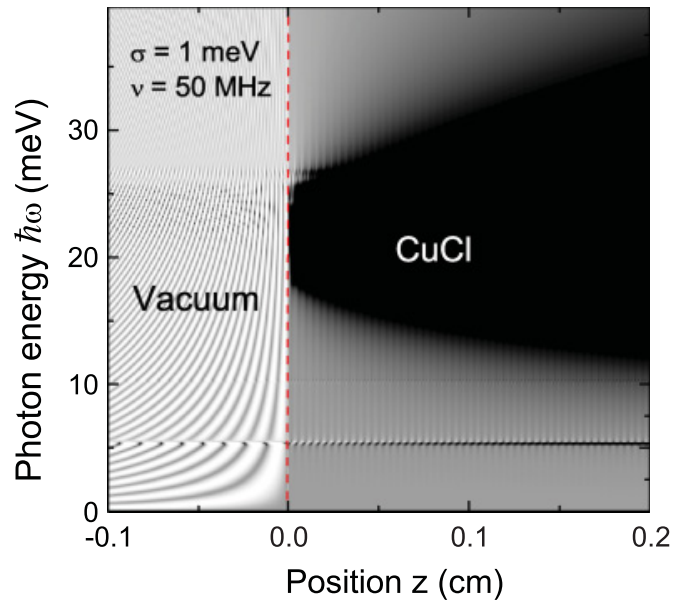

FIG. 13. (Color online) The electric field intensity $|E(z, \omega)|^{2}$ in vacuum and semiconductor regions, calculated for $\sigma=1 \mathrm{meV}, v=$ $50 \mathrm{MHz}, \gamma=0.2 \mathrm{meV}$, and different frequencies of the incoming terahertz light. Black in the logarithmic gray scale map corresponds to $|E|^{2} \rightarrow 0$.

semiconductor superlattices where the Bragg scattering can also be neglected.

A clear bump just above the Restrahlen band seen in the top spectrum in Fig. 11 which is calculated for a relatively low acoustic frequency is due to accumulation of band gaps near the bottom of the UP branch (a similar accumulation of gaps takes place at $\Omega \rightarrow \infty$ near the top of the LP branch; it has been already discussed in Sec. IV C). This narrow spectral region remains infrared-active in higher Bragg orders due to the existence of polaritons which have momenta the same order of magnitude as the acoustic wavevector $K$ (very small in this case) and thus can be scattered by such an AW.

\section{B. Electric field distribution and interaction length}

When the wavelength of the incident light and the period of the acoustic modulation are comparable as shown in Fig. 13, it results in significant spectral changes which are mainly due to a strong Bragg scattering of the polariton waves from the propagating periodic patterns induced by the AW. The electric field distribution in Fig. 13 corresponds to the terahertz spectrum shown in the top panel of Fig. 5(a). While in the vacuum region the modulations of the electric field are determined exclusively by the light wavelength (respectively $\omega)$ and are caused by a superposition of the incoming and outgoing waves. In the semiconductor region the period of modulations is the same for all $\omega$ and is given by the acoustic wavelength $2 \pi / K$. At around $\hbar \omega=5 \mathrm{meV}$ the AW produces a half- $\lambda$ modulation ( $\lambda$ is the wavelength of the light propagating inside a semiconductor) that results in a destructive interference of co- and contrapropagating polariton (light) waves and thus in quick attenuation of the electric field seen as a narrow black stripe in Fig. 13.

The decay length of the electric field gives us an upper bound for an estimate of the acousto-optic interaction length $l_{\text {int }}$. In resonant acousto-optics, such a length characterizes the scale in real space where the light effectively couples to the ultrasonic wave by means of the TO phonons. The interaction

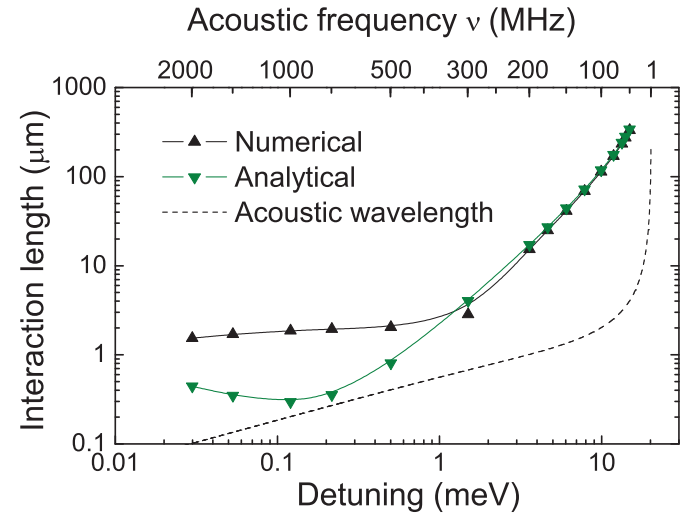

FIG. 14. (Color online) Interaction length, corresponding to the first acoustically induced LP band gap $\omega=\omega_{1}$, calculated analytically via Eq. (51) and evaluated numerically from the electric field profile, for $\sigma=1 \mathrm{meV}, \gamma=0.2 \mathrm{meV}$, and different values of the acoustic frequency (top axis) or the detuning $\omega_{t}-\omega_{1}$ (bottom axis). The dashed line shows the acoustic wavelength.

length calculated at the resonant frequency $\omega=\omega_{1}$ of the first AW-induced band gap is evaluated analytically in Appendix E, assuming $K l_{\text {int }} \ll 1$. Taking into account the finite TO-phonon damping $\gamma$, it takes the following form:

$$
l_{\text {int }}=\frac{K c^{2}}{\sigma \varepsilon_{b} \omega_{t}} \frac{\left(\omega_{t}^{2}-\omega^{2}\right)^{2}+4 \gamma^{2} \omega^{2}}{4 \omega^{2} \omega_{R}^{2}} .
$$

Figure 14 shows the interaction length numerically evaluated from the decay of the electric field at $\omega=\omega_{1}$ in the LP part of the spectrum. It also shows an excellent agreement with the analytic result Eq. (51) over the whole range of acoustic frequencies $\Omega$ or detuning $\delta \omega=\omega_{t}-\omega_{1}$. When decreasing the detuning, the interaction length also decreases monotonously and at very small values of $\delta \omega$ is limited by the TO-phonon damping. It exceeds the acoustic wavelength $2 \pi / K$ (also shown in Fig. 14 by a dashed line) by a few tens to hundred times only.

\section{CONCLUSIONS}

We have studied, both numerically and analytically, the far-infrared properties of acoustically pumped semiconductor crystals, in a geometry of collinear propagation of the light and ultrasound waves. Owing to the cubic phonon anharmonicity, TO phonons mediate the coupling between the light and the acoustic fields that results in drastic changes of the terahertz properties of semiconductors and in enhancement of the acousto-optic effect by several orders of magnitude compared to the conventional acousto-optics.

We have developed an efficient numerical method for calculation of acoustically induced band gaps, electric field distribution, and such infrared properties of semiconductors as transmission, reflection, and Bragg scattering of light. The method is based on the plane-wave expansion of the electric field and TO-phonon-induced polarization. It allows us to solve the coupled Maxwell's and material equations (including a phonon anharmonic source term due to a pumping AW) by mapping them into a linear matrix problem. We have provided a detailed analysis of the Bragg replicas of the incoming 
light (the Bragg replicas are frequency selected in the present case of collinear geometry) and demonstrated a one-to-one correspondence between the spikes in the reflectivity and the acoustically induced gaps in the polariton quasienergy spectrum. The gaps open at the energies $\omega_{N}$ satisfying the Bragg condition Eq. (48), manifest themselves as spikes in downconverted Bragg replicas $r_{n}$ with $n=-1,-2, \ldots$, $-N$, and correspond to $N$-phonon transitions (dressed with higher orders) between different polariton states in the crystal. In particular, the reflectivity is enhanced considerably when the acoustic wavelength is close to half integer $(N / 2)$ wavelength of the light in crystal. In this case a polariton wave is scattered by the periodic pattern of acoustic modulation of the TO-phonon field emitting $N$ acoustic phonons. The copropagating polariton wave and contrapropagating scattered wave interfere destructively to produce the electric field decay in the crystal. We have calculated the length of such decay which, in turn, limits the interaction length between the light and the ultrasound waves. The latter can be as short as only tens of the acoustic wavelengths, much shorter than in conventional acousto-optics.

Our analytic approach is based on the perturbation theory developed in the limit of weak coupling $\sigma / \omega_{t} \ll 1$ and allows us to evaluate and analyze the band-gap widths, the Bragg replicas, and the interaction length. Moreover, being in good quantitative agreement with numerical results, the analytic approach remains a reliable and accessible tool for calculation of the terahertz properties up to a moderate coupling strength ( $\sigma=1 \mathrm{meV}$ in $\mathrm{CuCl}$, corresponding to the acoustic intensity $I_{\mathrm{ac}}=14.8 \mathrm{~kW} / \mathrm{cm}^{2}$ ). We have also provided a refinement of our analytical method which uses an acoustically modified polariton dispersion and considerably improves the calculation of the sharp spikes in the reflectivity.

Finally, in the limits of very low and very high acoustic frequency $\Omega$, the contribution of all higher Bragg replicas $r_{n}$ with $n \neq 0$ vanishes, but the central replica $r_{0}$ still shows considerable spectral changes which grow dramatically with the acoustic intensity (or with $\sigma$ ). In these limiting cases, the wavelength of the propagating electromagnetics waves is either too small $(\Omega \rightarrow 0)$ or too large $(\Omega \rightarrow \infty)$ compared to the acoustic wavelength to feel the periodical modulation of the crystal; therefore, no Bragg scattering occurs. Instead, the polariton waves propagate as in a homogeneous medium which can be characterized by an $\omega$-dispersive effective dielectric constant.

\section{ACKNOWLEDGMENTS}

We thank S. G. Tikhodeev and R. Zimmermann for valuable discussions. This work was supported by the Royal Society (Grant No. JP0766306), EPSRC, and WIMCS.

\section{APPENDIX A: FOURIER TRANSFORM OF THE ELECTRIC FIELD AND POLARIZATION}

In this section, we show that the partial waves of the electric field and TO-phonon polarization, which are found as eigenfunctions of Eqs. (5) and (6), can be written in the form of a single Fourier transform [Eqs. (7) and (8)]. Due to the periodicity introduced by the AW, each partial wave can be expanded into Fourier series, both in time and in space. In problems similar to the quasienergy spectrum calculation where the wave vector $k$ is a fixed real-valued parameter, it is convenient to use the following space-time Fourier transform of a partial wave:

$$
\begin{aligned}
& E(z, t)=\sum_{n m} \mathcal{E}_{n m} e^{i(k+n K) z} e^{-i(\omega+n \Omega+m \Omega) t}, \\
& P(z, t)=\sum_{n m} \mathcal{P}_{n m} e^{i(k+n K) z} e^{-i(\omega+n \Omega+m \Omega) t} .
\end{aligned}
$$

Substituting the above expansions into Eqs. (5) and (6) and equating coefficients at the same exponentials results in the eigenvalue problem

$$
\begin{gathered}
{\left[(k+n K)^{2}-\frac{\varepsilon_{b}}{c^{2}}(\omega+n \Omega+m \Omega)^{2}\right] \mathcal{E}_{n m}} \\
=\frac{4 \pi}{c^{2}}(\omega+n \Omega+m \Omega)^{2} \mathcal{P}_{n m}, \\
{\left[\omega_{t}^{2}-2 i \gamma(\omega+n \Omega+m \Omega)-(\omega+n \Omega+m \Omega)^{2}\right] \mathcal{P}_{n m}} \\
+2 \sigma \omega_{t}\left(\mathcal{P}_{n-1, m}+\mathcal{P}_{n+1, m}\right)=\frac{\varepsilon_{b} \omega_{R}^{2}}{4 \pi} \mathcal{E}_{n m},
\end{gathered}
$$

which has to be solved to find eigenvalues $\omega$ and eigenvectors $\mathcal{E}_{n m}$ (and $\mathcal{P}_{n m}$ ). It is convenient, however, to introduce a new eigenvalue $\bar{\omega}=\omega+m \Omega$ which makes the eigenvalue problem $m$-independent, so that the eigenvectors $\mathcal{E}_{n m}$ and $\mathcal{E}_{n m^{\prime}}$ calculated for different $m$ and $m^{\prime}$ turn out to be proportional to each other and corresponding to the same, $m$-independent eigenvalue $\bar{\omega}$. In other words, all terms in Eqs. (A1) and (A2) having the same index $n$ are linearly dependent, which effectively eliminates the $m$ summation, bringing the above series to the form of Eqs. (7) and (8).

Note that the second index $m$ of the formal double Fourier transform $\mathcal{E}_{n m}$ and $\mathcal{P}_{n m}$ has nothing to do with the index $j$ in $E_{n j}$ and $P_{n j}$ used throughout the paper to label different partial waves (normalized polariton eigenstates), while the first index $n$ denotes different Fourier/Bragg components of the same partial wave.

\section{APPENDIX B: LINEARIZATION OF THE EIGENVALUE PROBLEM}

The eigenvalue problem given by Eqs. (9) and (10) is nonlinear, both in $\omega$ and in $k$, and is solved either to find the polariton eigenfrequencies in Sec. III A or to find the complex polariton wave vectors in Sec. IV A. Let us show that in both cases this set of equations can be reduced to a standard linear eigenvalue problem.

(i) Let us first consider the quasienergy dispersion $\omega(k)$ in which a real-valued wave vector $k$ is a given number (parameter). We assume $\gamma=0$ as done in Sec. III A. To linearize the eigenvalue problem, we introduce two new vectors,

$$
\begin{aligned}
& A_{n}=(\omega+\Omega) P_{n}, \\
B_{n}= & (\omega+\Omega)\left(\varepsilon_{b} E_{n}+4 \pi P_{n}\right) \\
= & (\omega+\Omega) \varepsilon_{b} E_{n}+4 \pi A_{n},
\end{aligned}
$$


and substitute them into Eqs. (9) and (10). The eigenvalue problem then takes the form

$$
\begin{gathered}
\omega P_{n}=-n \Omega P_{n}+A_{n}, \\
\omega E_{n}=-n \Omega E_{n}+\frac{B_{n}}{\varepsilon_{b}}-\frac{4 \pi A_{n}}{\varepsilon_{b}}, \\
\omega B_{n}=-n \Omega B_{n}+c^{2}(k+n K)^{2} E_{n}, \\
\omega A_{n}=-n \Omega A_{n}-\frac{\varepsilon_{b} \omega_{R}^{2}}{4 \pi} E_{n} \\
+\omega_{t}^{2} P_{n}+2 \sigma \omega_{t}\left(P_{n+1}+P_{n-1}\right) .
\end{gathered}
$$

The above set of equations can be written in a compact matrix form,

$$
\omega \overrightarrow{\mathbb{X}}=\hat{\mathbb{W}} \overrightarrow{\mathbb{X}}
$$

where $\hat{\mathbb{W}}$ and $\overrightarrow{\mathbb{X}}$ are, respectively, a $4 M \times 4 M$ matrix and a $4 M$ vector defined as (the truncation of the matrices the and the definition of $M$ are introduced in Sec. III A)

$$
\hat{\mathbb{W}}=\left|\begin{array}{cccc}
-\Omega \hat{n} & 0 & \omega_{t}^{2} \hat{1}+2 \sigma \omega_{t} \hat{f} & 0 \\
0 & -\Omega \hat{n} & 0 & c^{2}(k \hat{1}+K \hat{n})^{2} \\
\hat{1} & 0 & -\Omega \hat{n} & 0 \\
-4 \pi \varepsilon_{b}^{-1} \hat{1} & \varepsilon_{b}^{-1} \hat{1} & 0 & -\Omega \hat{n}
\end{array}\right|,
$$

$$
\overrightarrow{\mathbb{X}}=\left|\begin{array}{c}
\vec{A} \\
\vec{B} \\
\vec{P} \\
\vec{E}
\end{array}\right|,
$$

where $\hat{1}, \hat{n}$, and $\hat{f}$ are $M \times M$ matrices having the properties

$$
\begin{gathered}
(\hat{1} \vec{P})_{n}=P_{n}, \\
(\hat{n} \vec{P})_{n}=n P_{n}, \\
(\hat{f} \vec{P})_{n}=P_{n-1}+P_{n+1},
\end{gathered}
$$

and $\vec{A}, \vec{B}, \vec{P}$, and $\vec{E}$ are $M$ vectors.

(ii) In the opposite case of the eigenvalue problem to be solved for the complex-valued momentum $k$, keeping the real frequency $\omega$ constant, we do essentially the same. However, before introducing a new vector, we express the polarization $P_{n}$ from Eq. (10), which can be written as

$$
\sum_{m} G_{n m} P_{m}=E_{n}
$$

where

$$
\begin{aligned}
G_{n m}= & \frac{4 \pi}{\varepsilon_{b} \omega_{R}^{2}}\left\{\left[\omega_{t}^{2}-2 i \gamma(\omega+n \Omega)-(\omega+n \Omega)^{2}\right] \delta_{n m}\right. \\
& \left.+2 \sigma \omega_{t}\left(\delta_{n, m+1}+\delta_{n, m-1}\right)\right\} .
\end{aligned}
$$

Noting that $G_{n m}$ does not depend on $k$ (it depends on $\omega$ only), this matrix can be inverted, resulting in the following equation:

$$
\frac{4 \pi}{c^{2}}(\omega+n \Omega)^{2} P_{n}=\sum_{m} H_{n m} E_{m},
$$

where the matrix $\hat{H}$ has the form

$$
H_{n m}=\frac{4 \pi}{c^{2}}(\omega+n \Omega)^{2}\left(G^{-1}\right)_{n m} .
$$

Comparing Eqs. (9) and (B15) and also introducing a new vector $\vec{F}$ defined as

$$
F_{n}=\left(k+n K+\sqrt{\varepsilon_{b}} \frac{\omega+n \Omega}{c}\right) E_{n},
$$

a linearized matrix eigenvalue problem takes the form

$$
\hat{\mathbb{V}} \overrightarrow{\mathbb{Y}}=k \overrightarrow{\mathbb{Y}}
$$

where $\hat{\mathbb{V}}$ and $\overrightarrow{\mathbb{Y}}$ are, respectively, a $2 M \times 2 M$ matrix and a $2 M$ vector, defined as

$$
\begin{gathered}
\hat{\mathbb{V}}=\left|\begin{array}{cc}
-\hat{n} K-\sqrt{\varepsilon_{b} \frac{\omega \hat{1}+\hat{n} \Omega}{c}} & \hat{1} \\
\hat{H} & -\hat{n} K+\sqrt{\varepsilon_{b}} \frac{\omega \hat{1}+\hat{n} \Omega}{c}
\end{array}\right|, \\
\overrightarrow{\mathbb{Y}}=\left|\begin{array}{l}
\vec{E} \\
\vec{F}
\end{array}\right| .
\end{gathered}
$$

The procedure described in Sec. IV A to refine the polariton wave vectors $\tilde{\kappa}_{j \neq 0}$ using the best calculated central root $\varkappa(\omega)$ utilizes the translational property of the modified polariton dispersion [Eq. (24)] and the following linear interpolation between neighboring points in frequency, $\omega_{i} \leqslant \omega-j \Omega \leqslant \omega_{i+1}$ :

$$
\begin{aligned}
\tilde{\kappa}_{j}(\omega) & =j K+\varkappa(\omega-j \Omega) \\
& =\varkappa\left(\omega_{i}\right)+\frac{\varkappa\left(\omega_{i+1}\right)-\varkappa\left(\omega_{i}\right)}{\omega_{i+1}-\omega_{i}}\left(\omega-j \Omega-\omega_{i}\right) .
\end{aligned}
$$

\section{APPENDIX C: ANALYTIC EVALUATION OF THE BAND GAPS}

When calculating the band-gap widths $\Delta_{N}$ we have to take into account the effect of mixing (and anticrossing) of two polariton waves. One wave has the wave vector $k=k_{N}=N K / 2$ and refers to the $n=0$ replica; the other has the wave vector $k=k_{N}-N K=-N K / 2$ and refers to $n=-N$ component of the electric field. To take them into account in the lowest $(N$ th) order we truncate the tridiagonal matrix equation (14) to

$$
\left|\begin{array}{ccccc}
D_{0} & -\sigma & 0 & \ldots & 0 \\
-\sigma & D_{-1} & -\sigma & \ldots & 0 \\
0 & -\sigma & D_{-2} & \ldots & 0 \\
\vdots & \vdots & \vdots & \ddots & \vdots \\
0 & 0 & 0 & \ldots & D_{-N}
\end{array}\right| \cdot\left|\begin{array}{c}
\alpha_{0} E_{0} \\
\alpha_{-1} E_{-1} \\
\alpha_{-2} E_{-2} \\
\vdots \\
\alpha_{-N} E_{-N}
\end{array}\right|=0
$$

where $D_{n} \equiv D_{n}\left(k_{N}, \omega\right)$ and

$$
D_{n}(k, \omega)=\frac{1}{2 \omega_{t}}\left[\omega_{t}^{2}-\omega^{2}-\frac{\omega_{R}^{2}}{\left.(k / q)^{2}-1\right)}\right],
$$


neglecting $\gamma$ and $\Omega$ [cf. with Eq. (15)], and $q=\sqrt{\varepsilon_{b}} \omega / c$. We then solve Eq. (C1) and find small deviations of $\omega$ from the bare gap positions $\omega_{N}$ given by the equation $D_{0}\left(k_{N}, \omega_{N}\right)=0$. First of all, we find deviations of $D_{0}$ from zero by solving

$$
\operatorname{det}\left|\begin{array}{ccccccc}
D_{0} & -\sigma & 0 & \ldots & 0 & 0 & 0 \\
-\sigma & D_{-1} & -\sigma & \ldots & 0 & 0 & 0 \\
0 & -\sigma & D_{-2} & \ldots & 0 & 0 & 0 \\
\vdots & \vdots & \vdots & \ddots & \vdots & \vdots & \vdots \\
0 & 0 & 0 & \ldots & D_{-2} & -\sigma & 0 \\
0 & 0 & 0 & \ldots & -\sigma & D_{-1} & -\sigma \\
0 & 0 & 0 & \ldots & 0 & -\sigma & D_{0}
\end{array}\right|=0
$$

where the symmetry property $D_{s}=D_{N-s}$ is used. This results in the following quadratic equation for $D_{0}$ :

$$
D_{0}^{2} S_{11}-2 D_{0} \sigma^{2} S_{12}+\sigma^{4} S_{22}=0,
$$

where (for the fixed $N$ )

$$
S_{n m}=\operatorname{det}\left|\begin{array}{cccccc}
D_{-n} & -\sigma & 0 & \ldots & 0 & 0 \\
-\sigma & D_{-n-1} & -\sigma & \ldots & 0 & 0 \\
0 & -\sigma & D_{-n-2} & \ldots & 0 & 0 \\
\vdots & \vdots & \vdots & \ddots & \vdots & \vdots \\
0 & 0 & 0 & \ldots & D_{-m-1} & -\sigma \\
0 & 0 & 0 & \ldots & -\sigma & D_{-m}
\end{array}\right| .
$$

Noting that

$$
S_{12}^{2}-S_{11} S_{22}=\sigma^{4}\left(S_{23}^{2}-S_{22} S_{33}\right)=\cdots=\sigma^{2(N-2)},
$$

we find

$$
D_{0}\left(\omega_{N}^{ \pm}\right)=\frac{\sigma^{2} S_{12} \pm \sigma^{N}}{S_{11}}
$$

where the two values $\omega_{N}^{ \pm}$are the energy positions of the top and the bottom of the $N$ th gap. The gap width $\Delta_{N}=\left|\omega_{N}^{+}-\omega_{N}^{-}\right|$ is then proportional to

$$
D_{0}\left(\omega_{N}^{+}\right)-D_{0}\left(\omega_{N}^{-}\right)=\frac{2 \sigma^{N}}{S_{11}} \approx \frac{2 \sigma^{N}}{D_{-1}^{2} D_{-2}^{2} \cdots D_{-\left[\frac{N}{2}\right]}^{s}},
$$

where we have neglected all orders higher than $N$. In the last equation, $s=1$ and $\left[\frac{N}{2}\right]=\frac{N}{2}$ for even $N$, and $s=2$ and $\left[\frac{N}{2}\right]=\frac{N-1}{2}$ for odd $N$. Finally, the band gap $\Delta_{N}$ is evaluated using the linear approximation

$$
D_{0}\left(\omega_{N}^{+}\right)-\left.D_{0}\left(\omega_{N}^{-}\right) \approx\left(\omega_{N}^{+}-\omega_{N}^{-}\right) \frac{\partial D_{0}}{\partial \omega}\right|_{\omega=\omega_{N}},
$$

where the derivative is calculated and simplified to

$$
\frac{\partial D_{0}}{\partial \omega}=-\frac{\omega_{t}^{2} \omega_{R}^{2}+\left(\omega_{t}^{2}-\omega^{2}\right)^{2}}{\omega_{t} \omega \omega_{R}^{2}}
$$

using the condition $D_{0}\left(k_{n}, \omega\right)=0$ for $\omega=\omega_{N}$, taken on the bare polariton dispersion.

\section{APPENDIX D: BRAGG REPLICAS IN THE LIMIT OF SMALL PERTURBATIONS}

Substitution of the plane-wave expansion Eqs. (29) and (30) into the Maxwell's and material equations (5) and (6) results in the tridiagonal matrix equation for the electric field components

$$
\alpha_{n j} D_{n j} E_{n j}-\sigma\left(\alpha_{n+1, j} E_{n+1, j}+\alpha_{n-1, j} E_{n-1, j}\right)=0,
$$

where

$$
\begin{aligned}
D_{n j} & =D_{n}\left(\tilde{\kappa}_{j}, \omega\right), \\
\alpha_{n j} & =\alpha_{n}\left(\tilde{\kappa}_{j}, \omega\right),
\end{aligned}
$$

and $D_{n}$ and $\alpha_{n}$ are given by Eqs. (15) and (16), respectively. Equation (D1) is similar to Eq. (14) but now includes explicitly the index $j$ which refers to a specific partial polariton wave. Using the translation property Eq. (24) we obtain

$$
\begin{aligned}
D_{n, j+s}(\omega) & =D_{n+s, j}(\omega-s \Omega), \\
\alpha_{n, j+s}(\omega) & =\alpha_{n+s, j}(\omega-s \Omega),
\end{aligned}
$$

and then, neglecting the acoustic frequency $\Omega$, we are able to introduce universal vectors $\tilde{E}_{n}, \tilde{D}_{n}, \tilde{\alpha}_{n}$, and $\tilde{\kappa}_{n}$ :

$$
\begin{gathered}
\tilde{E}_{n+j}(\omega)=E_{n j}(\omega), \\
\tilde{D}_{n+j}(\omega)=D_{n j}(\omega), \\
\tilde{\alpha}_{n+j}(\omega)=\alpha_{n j}(\omega), \\
\tilde{\kappa}_{j}(\omega)=\varkappa(\omega)+j K,
\end{gathered}
$$

where $\varkappa(\omega)$ is the central root introduced in Eq. (28). Then Eq. (D1) takes the form

$$
\tilde{\alpha}_{n} \tilde{D}_{n} \tilde{E}_{n}-\sigma\left(\tilde{\alpha}_{n+1} \tilde{E}_{n+1}+\tilde{\alpha}_{n-1} \tilde{E}_{n-1}\right)=0,
$$

which is formally equivalent to Eq. (14) but has a different meaning. This is because it effectively couples components of different partial waves (having different $j$ ). In the following we drop all tildes in the above quantities for simplicity of notation.

To solve this equation perturbatively we note that $E_{n}=$ $O\left(\sigma^{|n|}\right)$. Neglecting higher orders in $\sigma$, we obtain from Eq. (D10)

$$
\begin{aligned}
\alpha_{1} D_{1} E_{1} & =\sigma \alpha_{0} E_{0} \\
\alpha_{2} D_{2} E_{2} & =\sigma \alpha_{1} E_{1} \\
& \cdots \\
\alpha_{ \pm N} D_{ \pm N} E_{ \pm N} & =\sigma \alpha_{ \pm(N-1)} E_{ \pm(N-1)}
\end{aligned}
$$

or, more explicitly,

$$
E_{ \pm N}=\sigma^{N} \frac{\alpha_{0}}{\alpha_{ \pm N}} \prod_{s=1}^{N} \frac{1}{D_{ \pm s}}
$$

where we have used the starting value $E_{0}=1$.

To calculate the reflection, we use the boundary conditions Eqs. (32) and (33), which take the form (also 
replacing $A_{j} \rightarrow A_{-j}$ )

$$
\begin{gathered}
\delta_{n, 0}+r_{n}=\sum_{j} A_{-j} E_{n+j}, \\
2 \delta_{n, 0}=\sum_{j} A_{-j} E_{n+j}\left(1+\beta_{n+j}\right),
\end{gathered}
$$

where

$$
\beta_{n}(\omega)=\frac{\varkappa(\omega)+n K}{q(\omega)}, \quad q(\omega)=\frac{\omega}{c} .
$$

In the perturbation approach, $A_{j}$ and $r_{n}$ have the same properties as $E_{n}$, namely, $A_{j}=O\left(\sigma^{|j|}\right)$ and $r_{n}=O\left(\sigma^{|n|}\right)$. We calculate them in a similar way, keeping only the leading-order terms,

$$
\begin{aligned}
A_{0} & =\frac{2}{1+\beta_{0}}, \\
A_{1} E_{0}\left(1+\beta_{0}\right) & =-A_{0} E_{1}\left(1+\beta_{1}\right), \\
A_{2} E_{0}\left(1+\beta_{0}\right) & =-A_{0} E_{2}\left(1+\beta_{2}\right)-A_{1} E_{1}\left(1+\beta_{1}\right), \\
\ldots & \\
A_{ \pm N} & =-\sum_{s=1}^{N} A_{ \pm(N-s)} E_{ \pm s} \frac{1+\beta_{ \pm s}}{1+\beta_{0}},
\end{aligned}
$$

as follows from Eq. (D14), and

$$
\begin{aligned}
r_{0}= & A_{0} E_{0}-1=\frac{1-\beta_{0}}{1+\beta_{0}}, \\
r_{ \pm N}= & \sum_{s=0}^{N} A_{ \pm(N-s)} E_{ \pm s}=\sum_{s=1}^{N} A_{ \pm(N-s)} E_{ \pm s}+A_{N} \\
= & \sum_{s=1}^{N} A_{ \pm(N-s)} E_{ \pm s} \frac{\beta_{0}-\beta_{ \pm s}}{1+\beta_{0}},
\end{aligned}
$$

as follows from Eqs. (D13) and (D16).

\section{APPENDIX E: ANALYTIC EVALUATION OF THE DECAY (INTERACTION) LENGTH}

To calculate the interaction length at the energy of the first acoustically induced band gap $\omega=\omega_{1}$, we take into account, in the resonant approximation, the contribution of only two co- and contrapropagating polariton waves. Keeping in Eq. (7) only $n=0$ and $n=-1$ terms,

$$
E(z, t)=e^{-i \omega t} l\left[E_{0} e^{i k z}+E_{-1} e^{i(k-K) z}\right],
$$

we evaluate the imaginary part $Q$ of the polariton wave vector

$$
k=\frac{K}{2}+i Q
$$

again, neglecting $\Omega$ and $\gamma$. Then Eq. (14) yields

$$
\begin{aligned}
& \alpha_{0} D_{0} E_{0}-\sigma \alpha_{-1} E_{-1}=0, \\
& \alpha_{-1} D_{-1} E_{-1}-\sigma \alpha_{0} E_{0}=0,
\end{aligned}
$$

from which we obtain

$$
D_{0} D_{-1}=\sigma^{2} \text {. }
$$

Using the property $D_{-1}(k, \omega)=D_{0}\left(k^{*}, \omega\right)=D_{0}^{*}(k, \omega)$ valid for the $k$ given by Eq. (E2) and introducing the real and imaginary parts of $D_{0}$ as $D_{0}=D_{0}^{\prime}+i D_{0}^{\prime \prime}$, Eq. (E4) transforms to

$$
D_{0}^{\prime 2}+D_{0}^{\prime \prime 2}=\sigma
$$

Since $D_{0}^{\prime}=0$ at the gap position $\omega=\omega_{1}$, the above equation results in

$$
D_{0}^{\prime \prime}\left(k, \omega_{1}\right)=\sigma^{2}
$$

or in a more explicit equation for $Q$ :

$$
\operatorname{Im} \frac{\omega_{R}^{2}}{\left(\frac{K / 2+i Q}{q}\right)^{2}-1}=2 \omega_{t} \sigma,
$$

where $q=\sqrt{\varepsilon_{b}} \omega / c$. Finally, assuming $K Q \ll 1$ leads to

$$
Q=\frac{2 \sigma \omega_{t}}{\omega_{R}^{2}} \frac{\left[(K / 2 q)^{2}-1\right]^{2}}{K / q^{2}}=\frac{2 \sigma \omega_{t} \omega_{R}^{2} q^{2}}{K\left(\omega_{t}^{2}-\omega^{2}\right)^{2}} .
$$

The interaction length is then evaluated from the decay of the electric field intensity and has the form

$$
l_{\text {int }}=\frac{1}{2 Q} \text {. }
$$

\footnotetext{
*On leave from General Physics Institute RAS, Moscow, Russia; egor.muljarov@astro.cf.ac.uk

${ }^{1}$ L. Brillouin, Ann. Phys. (Paris) 17, 88 (1922).

${ }^{2}$ P. Debye and F. W. Sears, Proc. Nat. Acad. Sci. USA 18, 409 (1932).

${ }^{3}$ R. Lucas and P. Biquard, J. Phys. Radium 3, 464 (1932).

${ }^{4}$ A. Korpel, Acousto-Optics (Dekker, New York, 1997).

${ }^{5}$ A. L. Ivanov and P. B. Littlewood, Phys. Rev. Lett. 87, 136403 (2001).

${ }^{6}$ K. Cho, K. Okumoto, N. I. Nikolaev, and A. L. Ivanov, Phys. Rev. Lett. 94, 226406 (2005).

${ }^{7}$ A. L. Ivanov, in Problems of Condensed Matter Physics, edited by A. L. Ivanov and S. G. Tikhodeev (Oxford University Press, Oxford, 2008), pp. 301-322.

${ }^{8}$ R. H. Poolman, E. A. Muljarov, and A. L. Ivanov, Phys. Rev. B 81, 245208 (2010).
}

${ }^{9}$ J. J. Hopfield, Phys. Rev. 112, 1555 (1958).

${ }^{10}$ M. M. de Lima Jr., R. Hey, P. V. Santos, and A. Cantarero, Phys. Rev. Lett. 94, 126805 (2005).

${ }^{11}$ M. M. de Lima Jr., M. van der Poel, P. V. Santos, and J. M. Hvam, Phys. Rev. Lett. 97, 045501 (2006).

${ }^{12}$ P. V. Santos, J. Appl. Phys. 89, 5060 (2001).

${ }^{13}$ M. M. de Lima Jr., R. Hey, and P. V. Santos, Appl. Phys. Lett. 83, 2997 (2003).

${ }^{14}$ M. M. de Lima Jr., P. V. Santos, R. Hey, and S. Krishnamurthy, Physica E 21, 809 (2004).

${ }^{15}$ S. Krishnamurthy and P. V. Santos, J. Appl. Phys. 96, 1803 (2004).

${ }^{16}$ M. M. de Lima Jr., and P. V. Santos, Rep. Prog. Phys. 68, 1639 (2005).

${ }^{17}$ N. Courjal, S. Benchabane, J. Dahdah, G. Ulliac, Y. Gruson, and V. Laude, Appl. Phys. Lett. 96, 131103 (2010). 
${ }^{18}$ R. A. Cowley, Rep. Prog. Phys. 31, 123 (1968).

${ }^{19}$ J. Menéndez and M. Cardona, Phys. Rev. B 29, 2051 (1984).

${ }^{20}$ K. Huang, Nature (London) 167, 779 (1951); Proc. R. Soc. London, Ser. A 208, 352 (1951).

${ }^{21}$ V. Romero-Rochin, R. M. Koehl, C. J. Brennan, and K. A. Nelson, J. Chem. Phys. 111, 3559 (1999).

${ }^{22}$ M. Wakaki, K. Kudo, and T. Shibuya, Physical Properties and Data of Optical Materials (CRC, New York, 2007).
${ }^{23}$ C. Ulrich, A. Gobel, K. Syassen, and M. Cardona, Phys. Rev. Lett. 82, 351 (1999).

${ }^{24}$ M. Cardona and T. Ruf, Solid State Commun. 117, 201 (2001).

${ }^{25}$ This symmetry property is fulfilled approximately provided that $\Omega \ll \omega$ and becomes strict assuming $v_{s}=0$. More generally, there is the following strict property: If $\omega(k)$ is an eigenvalue of Eqs. (9) and $(10)$, then $-\omega^{*}\left(-k^{*}\right)$ is also an eigenvalue.

${ }^{26}$ W. C. Tait, Phys. Rev. B 5, 648 (1972). 